\title{
Petrology of Peridotites and Related Gabbroic Rocks Around the Kairei Hydrothermal Field in the Central Indian Ridge
}

\author{
Tomoaki Morishita, Kentaro Nakamura, Takazo Shibuya, \\ Hidenori Kumagai, Taichi Sato, Kyoko Okino, Hiroshi Sato, \\ Rie Nauchi, Kaori Hara, and Ryo Takamaru
}

\begin{abstract}
Peridotites and related gabbroic rocks are widely exposed in the Central Indian Ridge, where the $\mathrm{H}_{2}$-rich-fluid-bearing Kairei hydrothermal field exists. We report on petrological and mineralogical characteristics of peridotites and gabbroic rocks recovered from an oceanic core complex at a latitude of $25^{\circ}$ South $\left(25^{\circ} \mathrm{S}\right.$ OCC $)$ and the Yokoniwa Rise around the Kairei hydrothermal field. Gabbros recovered from the $25^{\circ} \mathrm{S}$ OCC show a wide range of variations in terms of mineral chemistry and mineral assemblages (olivine-gabbro, gabbronorite to highly evolved oxide gabbro) and are similar to those from the Atlantis Bank of the Southwest Indian Ridge, an ultraslow-spreading ocean ridge. Peridotites recovered from $25^{\circ} \mathrm{S}$ OCC and the Yokoniwa Rise are generally characterized by moderately to highly depleted melt components. The partial melting of these peridotites is followed by chemical modification through interaction with a wide range of melts from relatively less evolved to highly evolved characteristics. Moderately to highly depleted melt components in the studied peridotites can be explained as being either residue after a relatively high-melt productivity period in intermediate-spreading ridges or a geochemically distinctive domain which has suffered from partial melting in the past rather than partial melting beneath the present mid-ocean ridge systems.
\end{abstract}

Keywords

Central Indian Ridge $•$ Gabbro $•$ Magmatism $•$ Peridotite

T. Morishita $(\bowtie)$

School of Natural System, College of Science and Engineering, Kanazawa University, Kakuma, Kanazawa 920-1192, Japan

K. Nakamura • T. Shibuya

H. Kumagai

Precambrian Ecosystem Laboratory (PEL), Japan Agency for Marine-Earth Science and Technology (JAMSTEC),

2-15 Natsushima, Yokosuka, Kanagawa 237-0061, Japan

T. Sato

Geological Survey of Japan, National Institute of Advanced Industrial Science and Technology, Central 7, 1-1-1, Higashi,

Tsukuba, Ibaraki 305-8567, Japan

\subsection{Introduction}

It is crucial to understand the generalities and the differences in ocean floor formations as a function of the spreading rate along the mid-ocean ridges within the context of plate

\footnotetext{
K. Okino

Atmosphere and Ocean Research Institute (AORI), The University of Tokyo, 5-1-5 Kashiwa, Chiba 277-8564, Japan

H. Sato

School of Business Administration, Senshu University,

2-1-1 Higashimita, Tama, Kawasaki, Kanagawa 214-8580, Japan

R. Nauchi $\bullet$ K. Hara $\bullet$ R. Takamaru

School of Natural System, College of Science and Engineering,

Kanazawa University, Kakuma, Kanazawa 920-1192, Japan
} 
tectonics. The southern end of the Central Indian Ridge (CIR) is characterized by an intermediate-spreading midocean ridge $(5 \mathrm{~cm} /$ year, full spreading rate) that is different from the Atlantic (slow-spreading) and the Pacific (fastspreading) ocean ridges. It is, therefore, the southern end of the CIR which will provide us with unique opportunities to study the magmatic and tectonic evolution along an intermediate mid-ocean ridge. One of the difficulties in regard to addressing these questions at oceanic spreading ridges is the paucity of in-situ samples from the seafloor.

Because of the discovery of a $\mathrm{H}_{2}$-rich-fluid-bearing hydrothermal field (Kairei hydrothermal field) (Hashimoto et al. 2001) coupled with its unique hydrothermal fluid chemistry and vent-endemic ecosystem (Gamo et al. 2001; Van Dover et al. 2001; Van Dover 2002; Takai et al. 2004; Gallant and Von Damm 2006; Kumagai et al. 2008) in the southern end of the CIR, three submersible expeditions of the SHINKAI 6500 and one dredge expedition of the Hakuho-maru were conducted around the Kairei hydrothermal field (Fig. 14.1). This study presents systematic data concerning the mineral compositions of the primary features of peridotites, now highly serpentinized/weathered, and related gabbroic rocks recovered from areas around the Kairei-hydrothermal field.

\subsection{Geological Background of the Studied Area}

The rate of spreading in the Central Indian Ridge (CIR) increases from slow-spreading of $30 \mathrm{~mm} / y e a r$ near the equator to intermediate-spreading with $48 \mathrm{~mm} /$ year at the Rodriguez Triple Junction (DeMets et al. 2010). The studied area is the southernmost CIR near the Rodriguez triple junction where three mid-ocean ridges (CIR, Southwestern Indian Ridge, Southeastern Indian Ridge) are intersected. A well-developed axial valley, segmented by fracture zones and non-transform discontinuities are identified in the southern part of the CIR. The ridge segments were identified from south to north, CIR-S1 and CIR-S2, respectively (Briais 1995). The ridge axis of CIR-1 is characterized as $7 \mathrm{~km}$ wide at the segment center with the widening to $12-15 \mathrm{~km}$ toward segment ends. The axial valley depth ranges from 3,600 to $4,400 \mathrm{~m}$. The ridge axis shows typical slowspreading ridge morphology, whereas off-axis morphology is rather chaotic. An oceanic core complex, $20 \times 10 \mathrm{~km}$ in size and 1,000 $\mathrm{m}$ higher than the adjacent seafloor, is located off-axis on the western flank of the CIR at a latitude of $25^{\circ}$ South $\left(25^{\circ} \mathrm{S}\right.$ OCC hereafter), between CIR-S1 and CIR-S2 (Mitchell et al. 1998; Kumagai et al. 2008; Morishita et al. 2009; Sato et al. 2009). The Kairei hydrothermal field is located on the eastern axial valley wall of CIR-S1 (Fig. 14.1). The Uraniwa Hills, where gabbros and dunites were mainly recovered from the top of the hill, is located off- axis, about $2 \mathrm{~km}$ to the east of the Kairei hydrothermal field (Nakamura et al. 2009). Petrogenesis of dunite-troctolite from the Uraniwa Hills will be shown in a separate paper. Serpentinized peridotites were already sampled at a small hill (Green Rock Hill and called as GRH hereafter), $15 \mathrm{~km}$ south of the $25^{\circ} \mathrm{S}$ OCC (Hellebrand et al. 2002) (Green Rock Hill in Fig. 14.1). Tectonic development of the studied area has been studied by several authors, but there is no clear picture yet (Briais 1995; Honsho et al. 1996; Mendel et al. 2000; Sato et al. 2009). Here we summarize petrological and mineralogical characteristics of peridotites and related gabbros from the $25^{\circ} \mathrm{S}$ OCC and the Kairei hydrothermal field (Yokoniwa Rise hereafter).

\subsection{Petrology}

Gabbros from the $25^{\circ} \mathrm{S}$ OCC are olivine gabbro, gabbro, gabbronorite (granular orthopyroxene-bearing gabbro) and oxide gabbro. The grain sizes of gabbros vary from doleritic fine-grained to coarse-grained rocks (Figs. 14.2 and 14.3). Most of the samples are mainly composed of plagioclase, clinopyroxene and amphibole. Accessory phases are olivine, magnetite, ilmenite, sulfides and apatite. Clinopyroxene usually occurs as a coarse grain (Fig. 14.3a), the interstitial phase interlocking with plagioclase (Fig. 14.3b) and sometimes forming oikocrysts. Orthopyroxene also occurs as a granular grain and is sometimes associated with clinopyroxene, forming a two-pyroxene aggregate (Fig. 14.3d). Olivine tends to occur in fine-grained samples or fine-grained parts of each sample showing variations in grain size within each sample (Fig. 14.4c). Brown and green amphiboles occur heterogeneously in most samples. Brown amphiboles are often gradually changed to green amphiboles, and probably have both igneous and metamorphic origins. Oxide minerals are frequently observed in some samples (Fig. 14.3c). The gabbros have magmatic textures, usually with no or very minor plastic deformation except for very localized millimeter-scale altered zones where green amphibole (tremolitic / actinolitic) and/or chlorite were formed. Chlorite veins and albite veins are also found in the altered zone. Olivine-bearing fine-grained doleritic gabbro cuts gabbronorite with inverted pigeonite (Fig. 14.4c). No troctolitic rocks were found in the $25^{\circ} \mathrm{S}$ OCC, whereas many troctolitic rocks were recovered from the Uraniwa Hills in the east of the Kairei hydrothermal field (Nakamura et al. 2009). One gabbro (919R09) is cut by a quartz-bearing leucocratic granitic network showing granophyric intergrowth of quartz and feldspar (Fig. 14.2). Amphiboles, both brown-colored and green-colored, are observed at the boundary between the granitic rock and the gabbro. Highly evolved silica-rich granitic rock was also reported from the $25^{\circ} \mathrm{S}$ OCC (Nakamura et al. 2007). 
Fig. 14.1 The sample localities of peridotites and related gabbros on bathymetry map around the south end of the Central Indian Ridge (CIR). The broken lines represent the estimated nontransform offsets (Okino et al. Chap. 11). The peridotite samples were recovered by the SHINKAI 6500 dives (star) and the R/V Hakuho-Maru dredges (circle). The red-filed star on the Hakuho Knoll is the locality of the Kairei Hydrothermal Field. The Uraniwa Hills (Kumagai et al. 2008), where troctolites and dunites were recovered, is also shown. The peridotite samples from the Green Rock Hill were reported by Hellebrand et al. (2002). RTJ Rodriguez Triple Junction
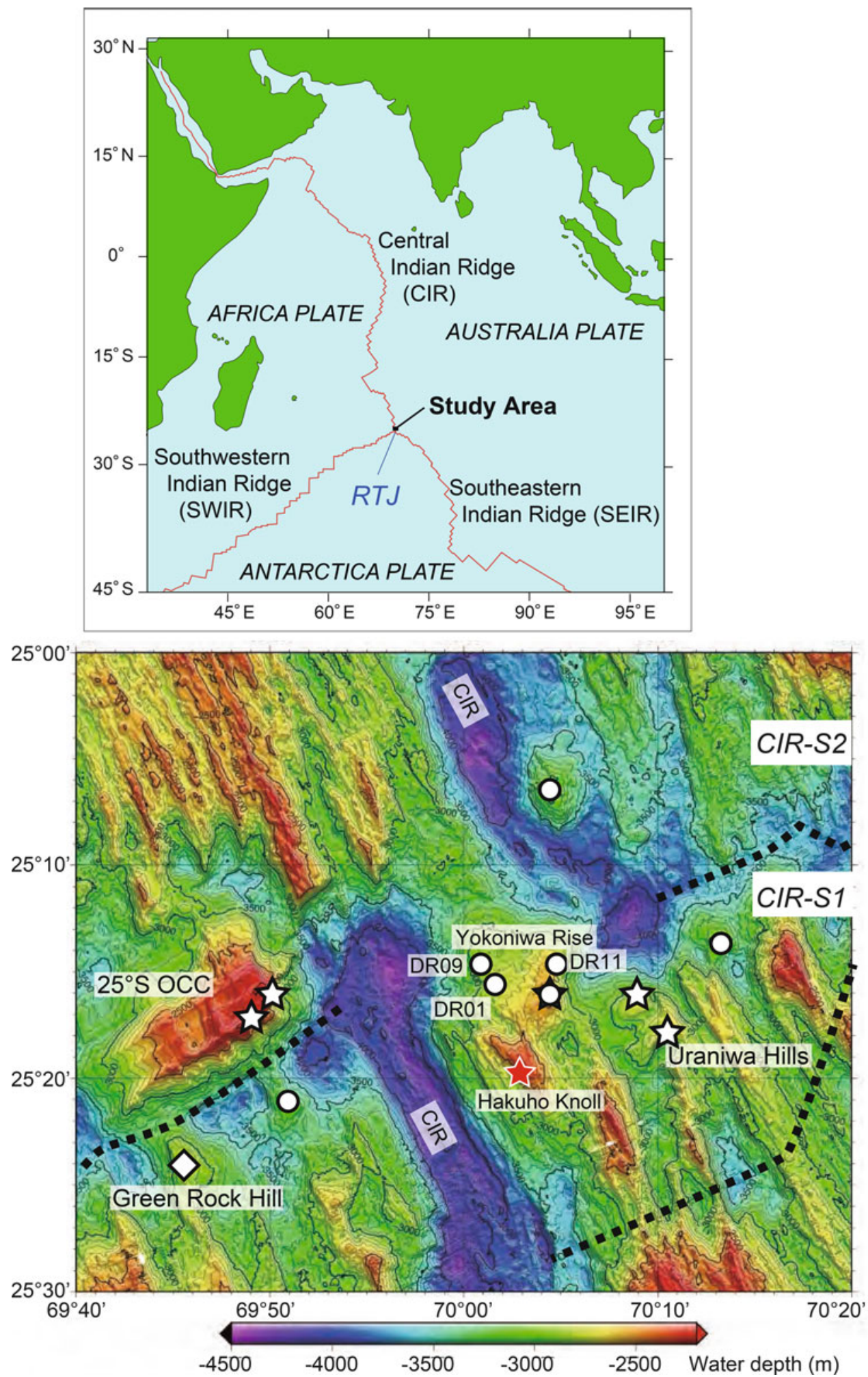

Peridotites sampled from the $25^{\circ} \mathrm{S}$ OCC are classified into two types: a granular peridotite (clinopyroxene-bearing harzburgite) recovered along the ridge-facing slope and a highly deformed type of rock recovered from the top surface of the $25^{\circ} \mathrm{S}$ OCC. The latter was formed by interaction between peridotite/gabbro rocks with hydrothermal fluids along the detachment fault (Morishita et al. 2009). Highly deformed samples as found in the $25^{\circ} \mathrm{S}$ OCC have never been recovered from the Yokoniwa Rise. Petrological characteristics of granular peridotites (clinopyroxene-bearing 

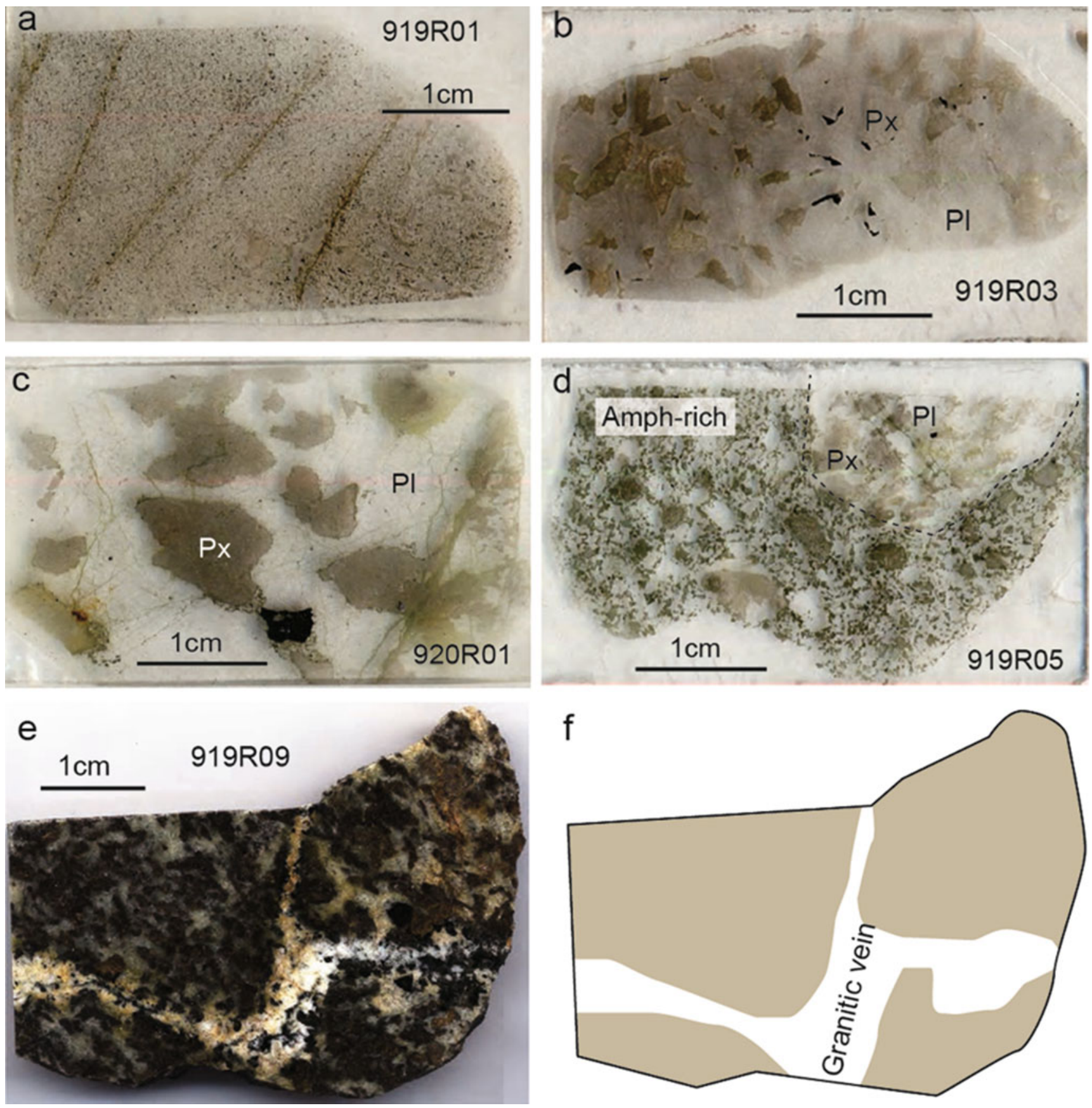

Fig. 14.2 Representative gabbro samples recovered from the $25^{\circ} \mathrm{S}$ OCC. (a) Fine-grained olivine gabbro (919R01). (b) Coarse-grained gabbronorite (919R03). (c) Coarse-grained gabbronorite (920R01). (d)

harzburgite to lherzolite) are basically the same between the $25^{\circ} \mathrm{S}$ OCC and the Yokoniwa Rise. Here we, therefore, briefly describe the petrological characteristics of granular peridotites from the $25^{\circ} \mathrm{S}$ OCC and the Yokoniwa Rise. Less deformed, granular to weakly deformed peridotites are extensively serpentinized and weathered, $>90 \%$ replacement of the primary minerals. Serpentinization and weathering result in variations in the color of samples, such as black, greenish

Heterogeneity in grain size showing amphibole-rich domain and gabbroic domain (919R05). (e) Quartz-bearing granitic network in gabbro (919R09) and its sketch (f). $P l$ plagioclase, $P x$ pyroxene

and oranges-like to the naked eye (Fig. 14.5). A part of some samples show white in color, where talc is frequently observed. Bastite textures with relics of orthopyroxene in the core are frequently found in samples. The cores of the orthopyroxene grains frequently contain exsolution lamellae of clinopyroxene. Several samples in both the $25^{\circ} \mathrm{S} \mathrm{OCC}$ and the Yokoniwa Rise were intruded by leucocratic veins (Fig. 14.5), up to $10 \mathrm{~mm}$ in thickness, mainly composed of 

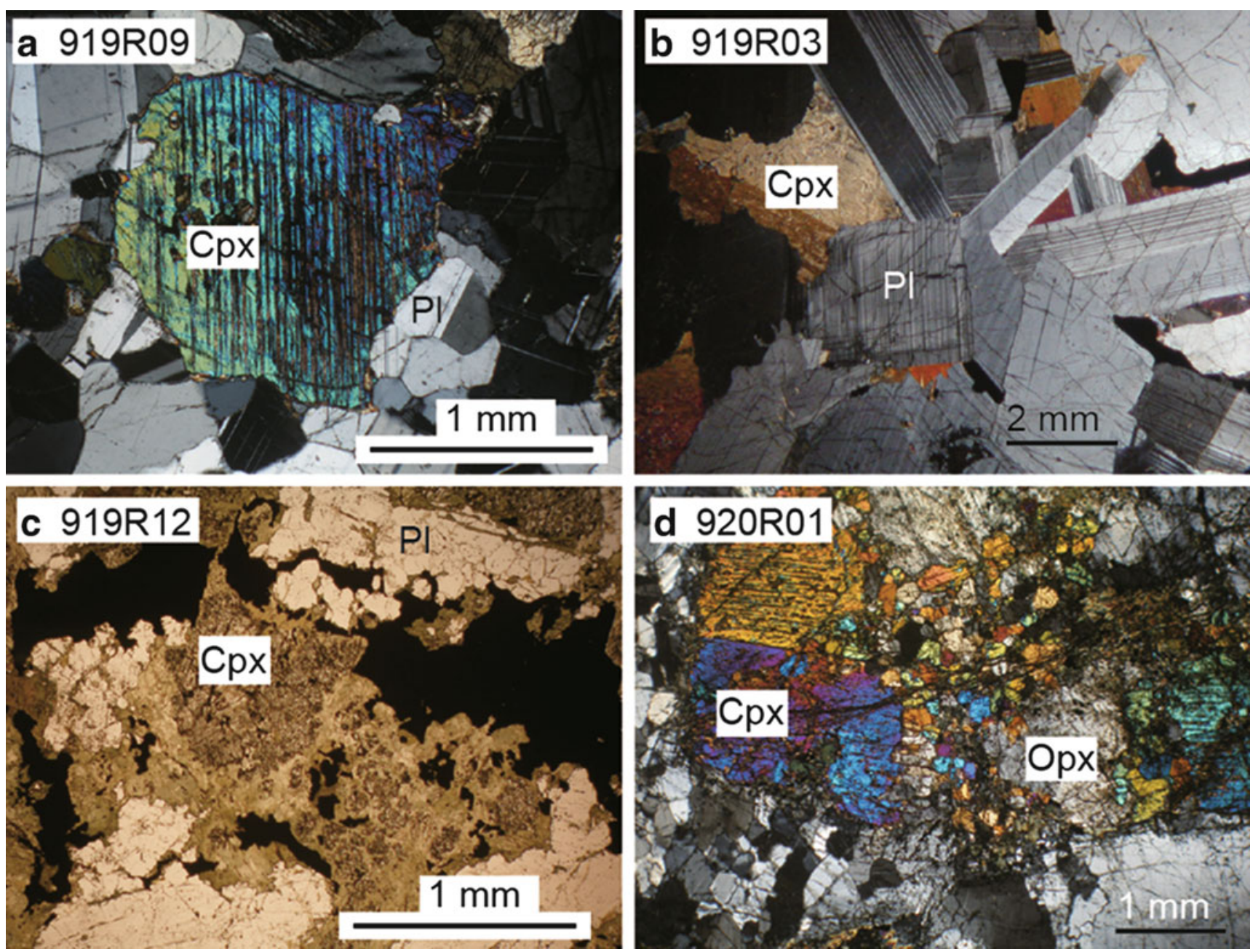

Fig. 14.3 Photomicroscope of gabbro samples recovered from the $25^{\circ} \mathrm{S}$ OCC. (a) Granular clinopyroxene (919R09) (Cross-polarized

(c) Oxide-rich gabbro (919R12) (Plane-polarized light), (d) Granular orthopyroxene associated with clinopyroxene (920R01) (Crosspolarized light). $P l$ plagioclase, $C p x$ clinopyroxene, $O p x$ orthopyroxene

chlorite and tremolite/actinolite with a small amount of ilmenite, zircon and apatite. The area near the thick leucocratic vein tends to be less serpentinized. Plagioclase, now completely altered, was observed in several samples (Fig. 14.5d). Spinel grains without altered plagioclase as well as altered plagioclase without spinel occur in the same thin section (Morishita et al. 2009).

\subsection{Mineral Chemistry}

\subsubsection{Analytical Method}

Major element compositions of minerals were analyzed by electron probe microanalysis (EPMA) with a JEOL JXA-8800 Superprobe system at Kanazawa University. The analyses were performed using an accelerating voltage of $15 \mathrm{kV}$ and beam current of $20 \mathrm{nA}$, using a $3 \mu \mathrm{m}$ diameter beam. Natural and synthetic mineral standards were employed for all minerals. JEOL software using ZAF corrections was employed (Morishita et al. 2003). We were generally attempting to obtain average compositions for the thin section. However, if the sample showed heterogeneity within each grain and/or each sample, single spot analyses were used as representative analytical data. The mineral chemistry of peridotites recovered from the $25^{\circ} \mathrm{S}$ OCC had already been described in a previous paper (Morishita et al. 2009). The mineral chemistry of peridotites recovered from the Yokoniwa Rise is similar to that from the $25^{\circ} \mathrm{S}$ OCC. Representative major element compositions of minerals of gabbroic rocks from the $25^{\circ} \mathrm{S}$ OCC and peridotites from the Yokoniwa Rise are shown in Tables 14.1, 14.2, 14.3, 14.4, 14.5, 14.6, 14.7, 14.8, and 14.9. 

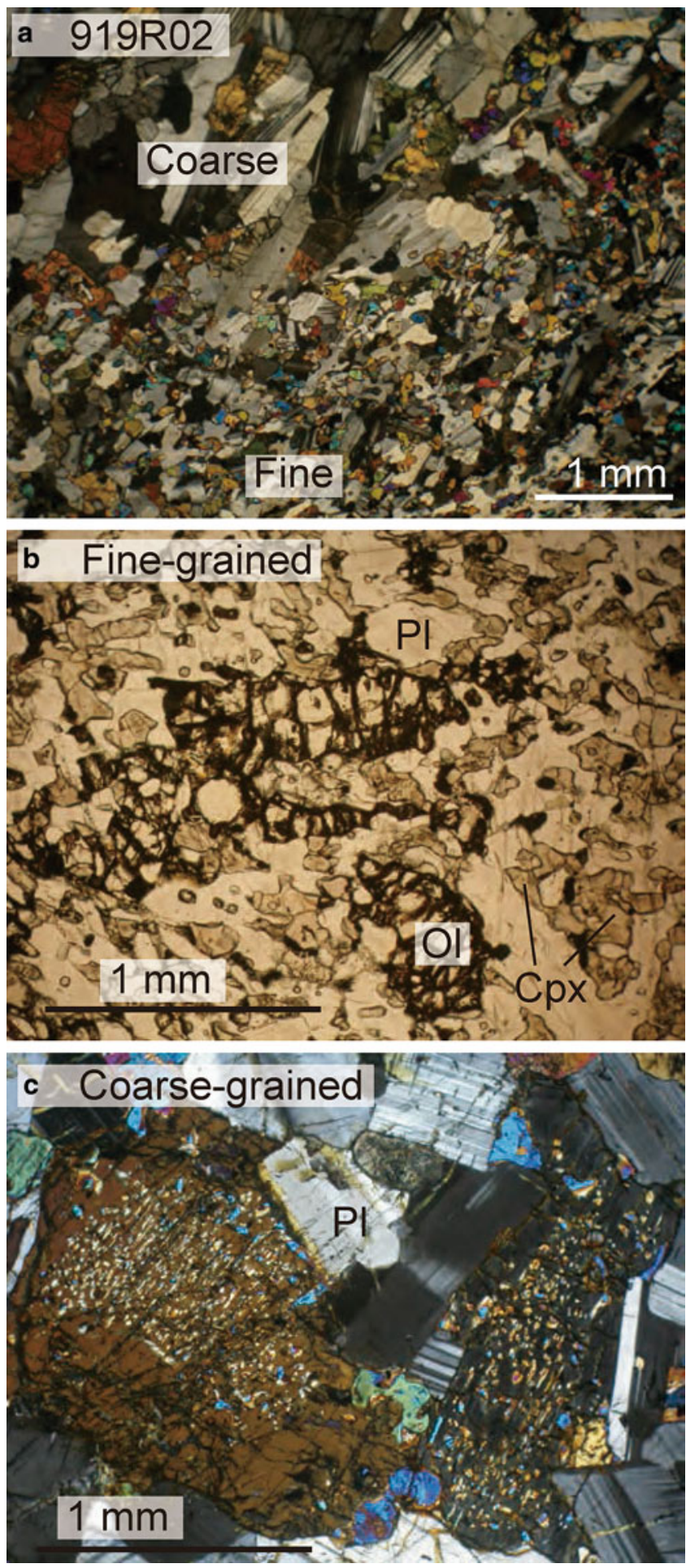

Fig. 14.4 Photomicroscope of heterogeneous gabbro (919R02). (a) Contact between fine-grained and coarse-grained parts (Crosspolarized light). (b) Fine-grained olivine gabbroic part (Plane-polarized light). (c) Coarse-grained part with inverted pegionite (Cross-polarized light).

\subsubsection{Gabbros from the $25^{\circ} \mathrm{S}$ OCC}

Mineral compositions in samples with variable grain sizes show different chemical compositions in each domain. The fine-grained part, which usually contains olivine, is higher in $\mathrm{Mg} \#\left(=\mathrm{Mg} /\left(\mathrm{Mg}+\mathrm{Fe}^{\text {total }}\right)\right.$ atomic ratio $)$ than the coarsegrained part within the sample as shown below. We, therefore, analyzed mineral compositions in each domain and describe them as different samples.

Olivine was found in three samples. The forsterite content of olivine is homogeneous within each sample and is 54,58 and 77, respectively (Table 14.1).

The $\mathrm{TiO}_{2}$ content of clinopyroxene is first increased with the decreasing of $\mathrm{Mg} \#$ until around 75 (Fig. 14.6a), as expected for the melt during crystallization of olivine, plagioclase and pyroxenes. The $\mathrm{TiO}_{2}$ content is then decreased (Fig. 14.6a), probably caused by the crystallization of Tibearing oxides, such as ilmenite and titanomagnetite (Table 14.2).

Plagioclase mostly shows normal zoning from the Carich core to the Na-rich rim. The anorthite content of coarsegrained plagioclase ranges from 37 to 59 in the core, whereas more Na-rich plagioclase, down to An6, was found in quartz-bearing granitic veins in gabbro as well as thin veins (albite vein in table) (Table 14.3). There is a good correlation between plagioclase compositions and lithologies, the Ca-rich plagioclase is usually found in olivine-bearing gabbro whereas the Na-rich plagioclase is found in oxide gabbro (Fig. 14.6).

The Mg\# of orthopyroxene core ranges from 67 to 76, which is slightly lower than ranges of $\mathrm{Mg} \#$ of clinopyroxene. The $\mathrm{TiO}_{2}$ content is usually lower than 0.5 wt.\%. The $\mathrm{Cr}_{2} \mathrm{O}_{3}$ content is low $<0.1 \mathrm{wt} . \%$ and is usually lower than the detection limit when the $\mathrm{Mg} \#$ is lower than 70 (Table 14.4).

Brown amphiboles are titaniferous pargastic hornblend (1-4 wt.\% $\left.\mathrm{TiO}_{2}\right)($ Table 14.5).

\subsubsection{Peridotites from the Yokoniwa Rise}

Olivine compositions are homogeneous in each sample. The forsterite content and the $\mathrm{NiO}$ content are within narrow compositional ranges with $90.8-91.3$ and $0.35-0.39$ wt.\%, respectively (Table 14.6).

The $\mathrm{Mg} \#$ of orthopyroxene is around 91. The $\mathrm{Al}_{2} \mathrm{O}_{3}$ content ranges from 2.5 to up to 4 wt. $\%$. The $\mathrm{TiO}_{2}$ content is lower than detection limit of analyses $(<0.06$ wt.\%) (Table 14.7). 

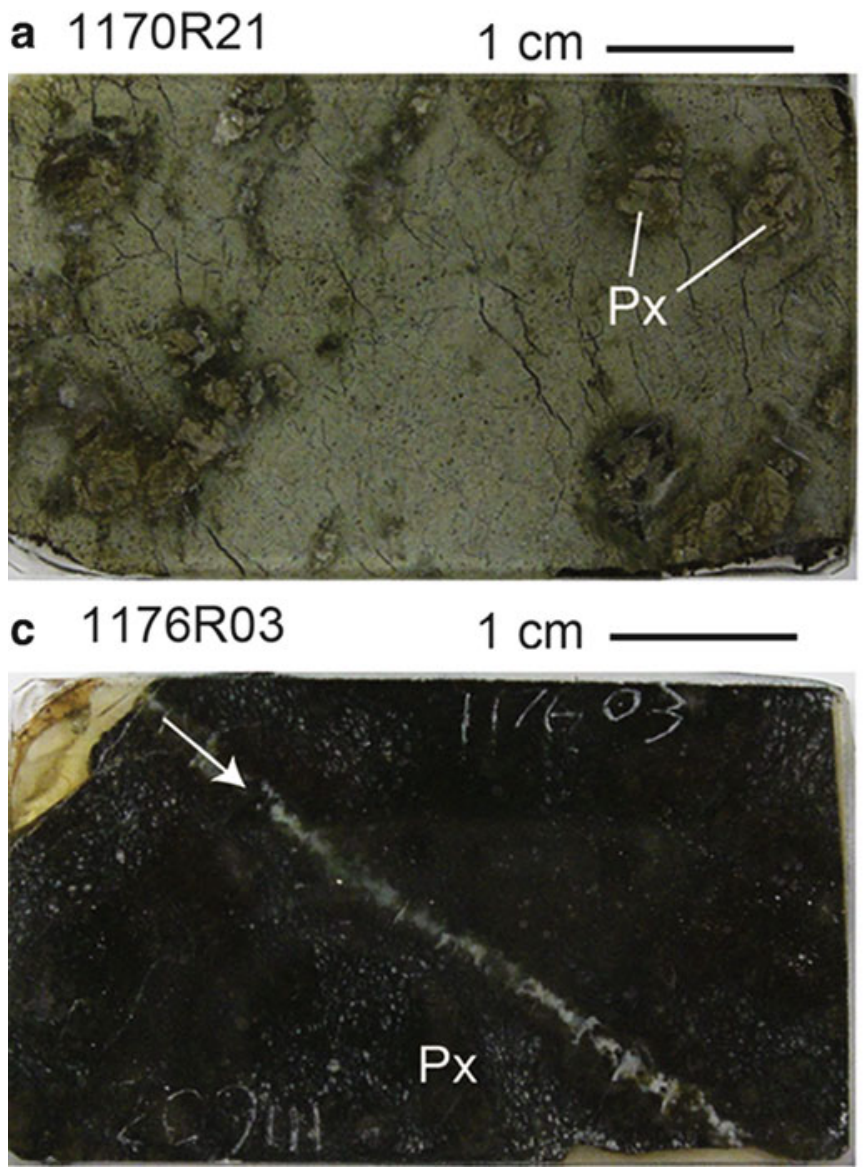
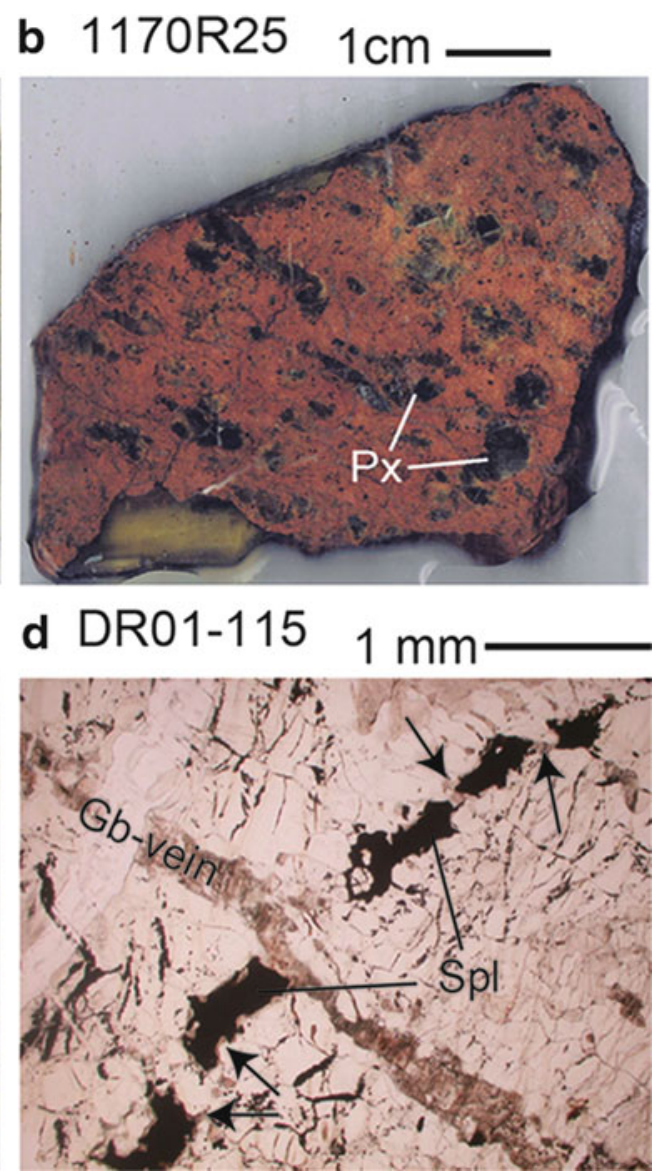

Fig. 14.5 Representative peridotite samples recovered from the Yokoniwa Rise. (a) Green-colored (1170R21) (upper panel), (b) brown-colored (middle panel) (1170R25) and (c) black-colored with thin leucocratic vein (indicated by arrow) (bottom panel) (1176R03). (d) Spinel trails cut by leucocratic vein (Gb-vein). Note that spinels are surrounded by plagioclase pseudomorph (indicated by arrows). Px pyroxene, $S p l$ spinel

Table 14.1 Representative major element compositions of olivine in gabbroic rocks from the $25^{\circ} \mathrm{S} \mathrm{OCC}$

\begin{tabular}{|c|c|c|c|c|c|c|c|c|c|}
\hline Sample & Section & $\mathrm{SiO}_{2}$ & $\mathrm{FeO}$ & $\mathrm{MnO}$ & $\mathrm{MgO}$ & $\mathrm{CaO}$ & $\mathrm{NiO}$ & Total & Fo \\
\hline \multirow[t]{2}{*}{ 919R01 } & Average & 35.30 & 36.20 & 0.48 & 28.43 & 0.06 & $<0.1$ & 100.6 & 58.3 \\
\hline & $\operatorname{STD}(\mathrm{N}=4)$ & 0.20 & 0.22 & 0.06 & 0.17 & $<0.03$ & - & 0.4 & 0.2 \\
\hline \multirow[t]{2}{*}{ 919R02 } & Average & 35.36 & 38.53 & 0.54 & 25.87 & 0.07 & $<0.1$ & 100.5 & 54.5 \\
\hline & $\operatorname{STD}(\mathrm{N}=2)$ & 0.23 & 0.03 & 0.09 & 0.23 & $<0.03$ & - & 0.4 & 0.2 \\
\hline \multirow[t]{2}{*}{ 919R05 } & Average & 38.93 & 21.27 & 0.32 & 39.39 & 0.05 & 0.14 & 100.1 & 76.7 \\
\hline & $\operatorname{STD}(\mathrm{N}=4)$ & 0.15 & 0.64 & 0.04 & 0.46 & $<0.03$ & 0.04 & 0.4 & 0.7 \\
\hline
\end{tabular}

The $\mathrm{Cr}_{2} \mathrm{O}_{3}, \mathrm{TiO}_{2}, \mathrm{Al}_{2} \mathrm{O}_{3}, \mathrm{Na}_{2} \mathrm{O}$ and $\mathrm{K}_{2} \mathrm{O}$ contents are mostly lower than detection limit of analyses, $<0.08$ wt. $\%,<0.06$ wt. $\%,<0.02$ wt. $\%$, $<0.03$ wt. $\%$ and $<0.03$ wt. $\%$, respectively. STD standard deviation, $N$ numbers of analyses, $F o$ forsterite content

Clinopyroxene in the samples with leucocratic veins are distinctively higher in $\mathrm{Na}_{2} \mathrm{O}\left(0.3-0.5\right.$ wt.\%) and $\mathrm{TiO}_{2}$ $(0.5-1.3$ wt.\%) content than those in vein-free samples, usually $<0.1 \mathrm{wt} . \%$ for both elements (Fig. 14.7).

Chemical compositions of spinels is homogeneous in samples without leucocratic veins, whereas spinels in samples with leucocratic veins show variations within and among grains (Table 14.9). The $\mathrm{Cr} \#$ and the $\mathrm{TiO}_{2}$ content of most samples are around 0.35 and $<0.1$ wt. $\%$, respectively, which are similar to the leucocratic vein-free sample in the $25^{\circ} \mathrm{S}$ OCC (Fig. 14.8). Both the $\mathrm{Cr} \#$ and the $\mathrm{TiO}_{2}$ content of spinels are high in the leucocratic vein-bearing samples and tend to be increased toward the leucocratic veins (Fig. 14.8). $\mathrm{YFe}^{3+}\left(=100 \mathrm{Fe}^{3+} /\left(\mathrm{Cr}+\mathrm{Al}+\mathrm{Fe}^{3+}\right)\right.$ atomic ratio $)$ is usually lower than 5 for homogeneous samples, and is relatively higher in high-Ti grains, up to 9 (Table 14.9). 
Table 14.2 Representative major element compositions of clinopyroxene in gabbroic rocks from the $25^{\circ} \mathrm{S}$ OCC

\begin{tabular}{|c|c|c|c|c|c|c|c|c|c|c|c|c|c|c|}
\hline Sample & Note & $\mathrm{SiO}_{2}$ & $\mathrm{TiO}_{2}$ & $\mathrm{Al}_{2} \mathrm{O}_{3}$ & $\mathrm{Cr}_{2} \mathrm{O}_{3}$ & $\mathrm{FeO}$ & $\mathrm{MnO}$ & $\mathrm{MgO}$ & $\mathrm{CaO}$ & $\mathrm{Na}_{2} \mathrm{O}$ & $\mathrm{K}_{2} \mathrm{O}$ & Total & $\mathrm{Mg} \#$ & $\mathrm{Cr} \#$ \\
\hline 919R01 & Core & 50.88 & 0.91 & 2.55 & 0.13 & 10.82 & 0.34 & 14.39 & 20.43 & 0.41 & $<0.03$ & 101.5 & 70.3 & 3.3 \\
\hline 919R02 (fine) & Core & 52.27 & 0.89 & 2.28 & 0.14 & 8.98 & 0.26 & 14.20 & 20.69 & 0.36 & $<0.03$ & 100.1 & 73.8 & 3.9 \\
\hline 919R02 (coarse) & Core & 51.70 & 0.74 & 1.84 & 0.16 & 11.29 & 0.28 & 13.54 & 20.14 & 0.39 & $<0.03$ & 100.1 & 68.1 & 5.4 \\
\hline 919R03 & Core & 53.01 & 0.91 & 2.61 & $<0.08$ & 9.15 & 0.28 & 14.40 & 20.96 & 0.53 & $<0.03$ & 101.9 & 73.7 & - \\
\hline 919R04 (fine) & Core & 53.08 & 0.62 & 2.35 & $<0.08$ & 8.35 & 0.23 & 14.81 & 21.51 & 0.39 & $<0.03$ & 101.4 & 76.0 & - \\
\hline 919R04 (coarse) & Core & 50.88 & 0.91 & 2.55 & 0.13 & 10.82 & 0.34 & 14.39 & 20.43 & 0.41 & $<0.03$ & 100.9 & 70.3 & 3.3 \\
\hline 919R05 (ol) & Core & 54.22 & 0.68 & 2.20 & 0.26 & 5.57 & 0.18 & 16.26 & 21.92 & 0.51 & $<0.03$ & 101.8 & 83.9 & 7.4 \\
\hline 919R05 (px) & Core & 54.02 & 0.71 & 2.17 & 0.09 & 6.79 & 0.19 & 16.52 & 20.64 & 0.32 & $<0.03$ & 101.5 & 81.3 & 2.7 \\
\hline 919R05 (amph) & Core & 53.17 & 0.91 & 2.64 & 0.15 & 8.39 & 0.23 & 14.78 & 20.67 & 0.73 & $<0.03$ & 101.7 & 75.8 & 3.6 \\
\hline 91R909 & Core & 51.96 & 0.83 & 2.42 & $<0.08$ & 7.16 & 0.23 & 15.69 & 21.07 & 0.39 & $<0.03$ & 99.8 & 79.6 & - \\
\hline 91R909 & Rim & 52.45 & 0.24 & 0.54 & $<0.08$ & 11.83 & 0.39 & 12.54 & 21.76 & 0.13 & $<0.03$ & 99.9 & 65.4 & - \\
\hline 919R12 & Core & 52.47 & 0.23 & 1.62 & $<0.08$ & 12.94 & 0.26 & 12.33 & 20.85 & 0.60 & 0.05 & 101.4 & 62.9 & - \\
\hline 919R12 & Rim & 53.17 & 0.07 & 0.38 & $<0.08$ & 12.05 & 0.37 & 12.56 & 22.46 & 0.34 & $<0.03$ & 101.4 & 65.0 & - \\
\hline 920R01 & Core & 51.95 & 0.79 & 2.39 & 0.09 & 9.68 & 0.30 & 14.92 & 21.23 & 0.41 & $<0.03$ & 101.8 & 73.3 & 2.3 \\
\hline 920R01 & Rim & 52.38 & 0.48 & 1.58 & $<0.08$ & 11.01 & 0.29 & 14.48 & 20.74 & 0.32 & $<0.03$ & 101.3 & 70.1 & - \\
\hline
\end{tabular}

The NiO content is mostly lower than $0.1 \mathrm{wt} . \%$. Fine fine-grained part, coarse coarse-grained part, ol olivine-bearing part, $p x$ olivine-free and amphibole-poor part, amph amphibole-rich part, $\mathrm{Mg} \# \mathrm{Mg} /\left(\mathrm{Mg}+\mathrm{Fe}^{\text {total }}\right)$ atomic ratio, $C r \# \mathrm{Cr} /(\mathrm{Cr}+\mathrm{Al})$ atomic ratio

Table 14.3 Representative major element compositions of plagioclase in gabbroic rocks from the $25^{\circ} \mathrm{S}$ OCC

\begin{tabular}{|c|c|c|c|c|c|c|c|c|c|c|c|}
\hline Sample & Note & $\mathrm{SiO}_{2}$ & $\mathrm{TiO}_{2}$ & $\mathrm{Al}_{2} \mathrm{O}_{3}$ & $\mathrm{FeO}$ & $\mathrm{MgO}$ & $\mathrm{CaO}$ & $\mathrm{Na}_{2} \mathrm{O}$ & $\mathrm{K}_{2} \mathrm{O}$ & Total & An \\
\hline 919R01 & Core & 54.60 & 0.08 & 29.35 & 0.37 & 0.04 & 11.71 & 5.34 & 0.06 & 101.6 & 54.8 \\
\hline 919R01 & Rim & 55.41 & $<0.05$ & 28.60 & 0.23 & 0.03 & 10.74 & 5.84 & 0.08 & 101.0 & 50.4 \\
\hline 919R02 (fine) & & 56.91 & $<0.05$ & 27.44 & 0.20 & 0.04 & 9.46 & 5.79 & 0.62 & 100.5 & 47.5 \\
\hline 919R02 (coarse) & & 59.18 & $<0.05$ & 25.80 & 0.47 & 0.10 & 7.95 & 7.34 & 0.10 & 101.0 & 37.4 \\
\hline 919R03 & Core & 56.92 & 0.10 & 26.93 & 0.37 & 0.10 & 9.73 & 6.16 & 0.08 & 100.3 & 46.6 \\
\hline 919R04 (fine) & Core & 55.60 & $<0.05$ & 28.33 & 0.28 & 0.04 & 11.10 & 5.69 & 0.03 & 101.1 & 51.9 \\
\hline 919R04 (fine) & Rim & 55.44 & $<0.05$ & 28.20 & 0.29 & 0.04 & 11.04 & 5.77 & $<0.03$ & 100.8 & 51.4 \\
\hline 919R04 (coarse) & Core & 57.15 & 0.14 & 27.40 & 0.32 & 0.03 & 10.01 & 6.18 & 0.08 & 101.2 & 47.2 \\
\hline 919R05 (ol) & & 54.50 & $<0.05$ & 28.80 & 0.13 & 0.03 & 12.20 & 4.81 & 0.04 & 100.5 & 58.3 \\
\hline 919R05 (px) & Core & 54.71 & $<0.05$ & 29.48 & 0.14 & 0.02 & 12.23 & 5.01 & 0.03 & 101.7 & 57.4 \\
\hline 919R05 (px) & Rim & 69.59 & $<0.05$ & 19.82 & 0.30 & $<0.02$ & 0.79 & 11.89 & $<0.03$ & 102.4 & 3.5 \\
\hline 919R05 (amph) & & 64.18 & $<0.05$ & 22.86 & 0.26 & $<0.02$ & 4.37 & 9.65 & 0.20 & 101.6 & 20.0 \\
\hline 919R09 & Core & 53.59 & 0.05 & 28.78 & 0.16 & 0.02 & 12.04 & 4.66 & 0.07 & 99.4 & 58.8 \\
\hline 919R09 & $\operatorname{Rim}$ & 52.87 & 0.05 & 29.31 & 0.20 & 0.05 & 12.35 & 4.40 & 0.08 & 99.4 & 60.8 \\
\hline 919R09 (granite) & & 60.86 & $<0.05$ & 24.00 & 0.16 & 0.02 & 6.08 & 8.21 & 0.18 & 99.5 & 29.1 \\
\hline 919R09 (granite) & & 67.19 & $<0.05$ & 20.16 & 0.00 & $<0.02$ & 1.25 & 11.11 & 0.07 & 99.9 & 5.9 \\
\hline 919R12 & Core & 59.27 & $<0.05$ & 25.69 & 0.14 & $<0.02$ & 7.96 & 7.48 & 0.16 & 100.7 & 37.0 \\
\hline 920R01 & Core & 56.60 & $<0.05$ & 27.68 & 0.41 & 0.04 & 10.31 & 5.93 & 0.03 & 101.0 & 49.0 \\
\hline 920R01 (ab vein) & & 66.80 & $<0.05$ & 21.00 & 0.00 & $<0.02$ & 1.62 & 10.95 & 0.03 & 100.5 & 7.5 \\
\hline 920R10 & Core & 57.29 & 0.06 & 26.49 & 0.11 & $<0.02$ & 8.85 & 6.95 & 0.12 & 99.9 & 41.3 \\
\hline 921R24 & Core & 66.77 & $<0.05$ & 20.68 & 0.29 & $<0.02$ & 1.56 & 11.49 & $<0.03$ & 100.8 & 7.0 \\
\hline
\end{tabular}

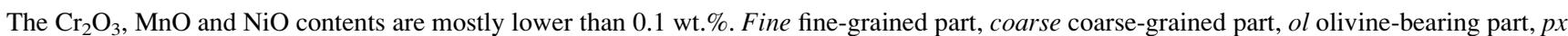
olivine-free and amphibole-poor part, amph amphibole-rich part, granite granitic vein, ab vein albite vein. An anorthite content $(100 \mathrm{Ca} /(\mathrm{Ca}+\mathrm{Na})$ $\mathrm{s}$ atomic ratio) 
Table 14.4 Representative major element compositions of orthopyroxene in gabbroic rocks from the $25^{\circ} \mathrm{S}$ OCC

\begin{tabular}{llllrrrrrrrrrrr}
\hline Sample & Note & $\mathrm{SiO}_{2}$ & $\mathrm{TiO}_{2}$ & $\mathrm{Al}_{2} \mathrm{O}_{3}$ & $\mathrm{Cr}_{2} \mathrm{O}_{3}$ & \multicolumn{1}{l}{$\mathrm{FeO}$} & $\mathrm{MnO}$ & $\mathrm{MgO}$ & $\mathrm{CaO}$ & $\mathrm{Na}_{2} \mathrm{O}$ & $\mathrm{K}_{2} \mathrm{O}$ & $\mathrm{Total}$ & $\mathrm{Mg \#}$ & $\mathrm{Cr \#}$ \\
\hline 919R01 & Core & 53.22 & 0.56 & 1.31 & $<0.08$ & 20.16 & 0.48 & 23.45 & 2.15 & $<0.03$ & $<0.03$ & 101.4 & 67.5 & - \\
\hline 919R02(coarse) & Core & 53.66 & 0.49 & 1.18 & $<0.08$ & 19.61 & 0.42 & 23.10 & 2.63 & 0.06 & $<0.03$ & 101.2 & 67.7 & - \\
\hline 919R04 (fine) & Core & 54.88 & 0.36 & 0.99 & $<0.08$ & 16.80 & 0.44 & 25.91 & 1.11 & $<0.03$ & 0.03 & 100.6 & 73.3 & - \\
\hline 919R04 (coarse) & Core & 53.66 & 0.49 & 1.18 & $<0.08$ & 19.61 & 0.42 & 23.10 & 2.63 & 0.06 & $<0.03$ & 101.2 & 67.7 & - \\
\hline 919R95 (ol) & & 55.48 & 0.29 & 1.13 & 0.11 & 13.88 & 0.34 & 27.94 & 1.10 & $<0.03$ & $<0.03$ & 100.3 & 78.2 & 6.3 \\
\hline 919R05(amph) & & 54.87 & 0.25 & 0.80 & $<0.08$ & 20.39 & 0.51 & 23.57 & 1.27 & $<0.03$ & $<0.03$ & 101.7 & 67.3 & - \\
\hline 919R09 & & 53.99 & 0.39 & 1.03 & $<0.08$ & 15.27 & 0.29 & 26.61 & 1.64 & 0.03 & $<0.03$ & 99.3 & 75.6 & - \\
\hline
\end{tabular}

The $\mathrm{NiO}$ content is mostly lower than $0.1 \mathrm{wt} . \%$. Note that the $\mathrm{CaO}$ content might be high because of inevitable contaminations from fine-grained clinopyroxene lamellae. Fine fine-grained part, coarse coarse-grained part, ol olivine-bearing part, $p x$ olivine-free and amphibole-poor part, amph amphibole-rich part, granite granitic vein, ab vein albite vein, $M g \# \mathrm{Mg} /\left(\mathrm{Mg}+\mathrm{Fe}^{\text {total }}\right)$ atomic ratio, $C r \# \mathrm{Cr} /(\mathrm{Cr}+\mathrm{Al})$ atomic ratio

Table 14.5 Representative major element compositions of brown amphibole in gabbroic rocks from the $25^{\circ} \mathrm{S}$ OCC

\begin{tabular}{|c|c|c|c|c|c|c|c|c|c|c|c|c|c|c|}
\hline Sample & Note & $\mathrm{SiO}_{2}$ & $\mathrm{TiO}_{2}$ & $\mathrm{Al}_{2} \mathrm{O}_{3}$ & $\mathrm{Cr}_{2} \mathrm{O}_{3}$ & $\mathrm{FeO}$ & $\mathrm{MnO}$ & $\mathrm{MgO}$ & $\mathrm{CaO}$ & $\mathrm{Na}_{2} \mathrm{O}$ & $\mathrm{K}_{2} \mathrm{O}$ & Total & $\mathrm{Mg \#}$ & $\mathrm{Cr} \#$ \\
\hline 919R01 & Opq & 42.81 & 2.57 & 10.50 & $<0.08$ & 15.08 & 0.20 & 12.48 & 11.13 & 2.61 & 0.16 & 97.6 & 0.596 & - \\
\hline 919R01 & Fine & 44.51 & 1.36 & 9.89 & 0.12 & 14.01 & 0.26 & 13.80 & 11.24 & 2.55 & 0.12 & 97.9 & 0.637 & 0.823 \\
\hline 919R02 (fine) & $P x$ & 43.96 & 3.44 & 10.50 & 0.61 & 11.54 & 0.11 & 13.62 & 10.88 & 2.84 & 0.13 & 97.6 & 0.678 & 3.739 \\
\hline 919R02 (fine) & Bleb & 43.21 & 4.03 & 10.29 & $<0.08$ & 14.53 & 0.18 & 11.30 & 11.11 & 2.98 & 0.23 & 97.9 & 0.581 & - \\
\hline 919R02 (fine) & Core & 45.63 & 1.84 & 8.17 & $<0.08$ & 18.56 & 0.23 & 10.75 & 10.73 & 2.15 & 0.31 & 98.4 & 0.508 & - \\
\hline 919R02 (coarse) & Core & 46.33 & 1.55 & 8.61 & $<0.08$ & 16.25 & 0.19 & 12.21 & 10.74 & 2.34 & 0.09 & 98.4 & 0.572 & - \\
\hline 919R02 (coarse) & Bleb & 43.64 & 4.16 & 11.13 & $<0.08$ & 11.26 & 0.12 & 13.47 & 11.18 & 3.35 & 0.12 & 98.4 & 0.681 & - \\
\hline 919R03 & Bleb & 46.76 & 1.10 & 9.28 & $<0.08$ & 11.98 & 0.16 & 14.55 & 11.23 & 2.33 & 0.17 & 97.6 & 0.684 & - \\
\hline 919R05 (amph) & $\mathrm{Pl}$ & 44.71 & 2.83 & 10.12 & 0.15 & 10.92 & 0.18 & 14.45 & 11.20 & 3.16 & 0.14 & 97.9 & 0.702 & 1.003 \\
\hline 919R05(amph) & Inter & 46.41 & 2.29 & 8.95 & 0.54 & 6.75 & 0.12 & 17.38 & 11.44 & 2.76 & 0.17 & 96.9 & 0.821 & 3.892 \\
\hline 919R09 & Inter & 43.46 & 3.44 & 11.42 & $<0.08$ & 8.76 & 0.08 & 15.14 & 11.54 & 2.89 & 0.16 & 97.0 & 0.755 & - \\
\hline 919R09 & $P x$ & 42.78 & 3.89 & 11.11 & 0.11 & 8.87 & 0.19 & 14.97 & 11.61 & 2.87 & 0.14 & 96.6 & 0.750 & 0.630 \\
\hline 919R12 & $P x$ & 43.72 & 2.67 & 9.62 & $<0.08$ & 17.34 & 0.32 & 10.88 & 10.82 & 2.83 & 0.24 & 98.5 & 0.528 & - \\
\hline
\end{tabular}

The $\mathrm{NiO}$ content is mostly lower than $0.1 \mathrm{wt} . \%$. Fine fine-grained part, coarse coarse-grained part, amph amphibole-rich part, opq around opaque mineral, $p x$ around pyroxene grain, $p l$ around plagioclase, inter interstitial grain, $M g \# \mathrm{Mg} /\left(\mathrm{Mg}+\mathrm{Fe}{ }^{\text {total }}\right)$ atomic ratio, $C r \# \mathrm{Cr} /(\mathrm{Cr}+\mathrm{Al})$ atomic ratio

Table 14.6 Representative major element compositions of olivine in peridotites from the Yokoniwa Rise

\begin{tabular}{|c|c|c|c|c|c|c|c|c|c|}
\hline Sample & Note & $\mathrm{SiO}_{2}$ & $\mathrm{FeO}$ & $\mathrm{MnO}$ & $\mathrm{MgO}$ & $\mathrm{CaO}$ & $\mathrm{NiO}$ & Total & Fo \\
\hline \multirow[t]{2}{*}{ 1170R01 } & Average & 40.92 & 8.55 & 0.12 & 50.49 & 0.04 & 0.38 & 100.5 & 91.3 \\
\hline & $\operatorname{STD}(\mathrm{N}=3)$ & 0.36 & 0.03 & 0.01 & 0.64 & $<0.03$ & 0.01 & 0.9 & 0.1 \\
\hline \multirow[t]{2}{*}{ 1170R04 } & Average & 40.75 & 9.03 & 0.13 & 50.33 & 0.04 & 0.37 & 100.6 & 90.9 \\
\hline & $\operatorname{STD}(\mathrm{N}=6)$ & 0.39 & 0.07 & 0.02 & 0.34 & $<0.03$ & 0.01 & 0.7 & 0.1 \\
\hline \multirow[t]{2}{*}{ 1170R09 } & Average & 41.11 & 9.04 & 0.13 & 50.31 & $<0.03$ & 0.38 & 101.2 & 90.8 \\
\hline & $\operatorname{STD}(\mathrm{N}=13)$ & 0.43 & 0.09 & 0.02 & 0.34 & $<0.03$ & 0.02 & 0.7 & 0.1 \\
\hline \multirow[t]{2}{*}{$1170 \mathrm{R} 21$} & Average & 41.37 & 8.78 & 0.13 & 50.63 & 0.03 & 0.39 & 101.4 & 91.1 \\
\hline & $\operatorname{STD}(\mathrm{N}=7)$ & 0.27 & 0.06 & 0.03 & 0.38 & $<0.03$ & 0.01 & 0.6 & 0.1 \\
\hline \multirow[t]{2}{*}{ 1170R25 } & Average & 41.10 & 8.85 & 0.12 & 50.43 & 0.05 & 0.37 & 101.0 & 91.0 \\
\hline & $\operatorname{STD}(\mathrm{N}=9)$ & 0.17 & 0.18 & 0.03 & 0.42 & 0.01 & 0.22 & 0.7 & 0.1 \\
\hline \multirow[t]{2}{*}{ 1170R27 } & Average & 41.16 & 8.92 & 0.13 & 50.24 & $<0.03$ & 0.37 & 100.8 & 90.9 \\
\hline & $\operatorname{STD}(\mathrm{N}=20)$ & 0.29 & 0.13 & 0.01 & 0.48 & $<0.03$ & 0.02 & 0.7 & 0.1 \\
\hline \multirow[t]{2}{*}{$1170 \mathrm{R} 28$} & Average & 41.25 & 8.93 & 0.13 & 50.28 & 0.06 & 0.37 & 101.1 & 90.9 \\
\hline & $\operatorname{STD}(\mathrm{N}=11)$ & 0.33 & 0.20 & 0.04 & 0.31 & $<0.03$ & 0.04 & 0.6 & 0.2 \\
\hline \multirow[t]{2}{*}{ 1176R15 } & Average & 40.77 & 8.68 & 0.13 & 49.30 & $<0.03$ & 0.35 & 99.3 & 91.0 \\
\hline & $\operatorname{STD}(\mathrm{N}=5)$ & 1.14 & 0.08 & 0.02 & 1.12 & $<0.03$ & 0.02 & 2.2 & 0.2 \\
\hline \multirow[t]{2}{*}{ 1176R20 } & Average & 41.25 & 8.84 & 0.13 & 50.38 & 0.03 & 0.37 & 101.0 & 91.0 \\
\hline & $\operatorname{STD}(\mathrm{N}=28)$ & 0.31 & 0.14 & 0.02 & 0.41 & $<0.03$ & 0.02 & 0.7 & 0.1 \\
\hline \multirow[t]{2}{*}{ DR01-106 } & Average & 41.15 & 9.07 & 0.15 & 50.55 & 0.03 & 0.39 & 101.4 & 90.9 \\
\hline & $\operatorname{STD}(\mathrm{N}=9)$ & 0.36 & 0.22 & 0.02 & 0.48 & $<0.03$ & 0.02 & 0.9 & 0.2 \\
\hline \multirow[t]{2}{*}{ DR11-003 } & Average & 41.60 & 9.06 & 0.12 & 50.28 & 0.05 & 0.36 & 100.6 & 90.8 \\
\hline & $\operatorname{STD}(\mathrm{N}=7)$ & 0.57 & 0.14 & 0.01 & 0.53 & $<0.03$ & 0.02 & 1.0 & 0.2 \\
\hline
\end{tabular}

The $\mathrm{Cr}_{2} \mathrm{O}_{3}, \mathrm{TiO}_{2}, \mathrm{Al}_{2} \mathrm{O}_{3}, \mathrm{Na}_{2} \mathrm{O}$ and $\mathrm{K}_{2} \mathrm{O}$ contents are mostly lower than detection limit of analyses, $<0.08$ wt. $\%,<0.06$ wt.\%, $<0.02$ wt.\%, $<0.03$ wt. $\%$ and $<0.03$ wt. $\%$, respectively. STD standard deviation, $N$ numbers of analyses, $F o$ forsterite content 
Table 14.7 Representative major element compositions of orthopyroxene in peridotites from the Yokoniwa Rise

\begin{tabular}{|c|c|c|c|c|c|c|c|c|c|c|c|c|c|}
\hline Sample & Note & $\mathrm{SiO}_{2}$ & $\mathrm{TiO}_{2}$ & $\mathrm{Al}_{2} \mathrm{O}_{3}$ & $\mathrm{Cr}_{2} \mathrm{O}_{3}$ & $\mathrm{FeO}$ & $\mathrm{MnO}$ & $\mathrm{MgO}$ & $\mathrm{CaO}$ & $\mathrm{NiO}$ & Total & Mg\# & $\mathrm{Cr} \#$ \\
\hline 1170R04 & Core & 55.64 & $<0.05$ & 3.36 & 0.96 & 6.00 & 0.13 & 33.46 & 0.89 & 0.10 & 100.6 & 0.909 & 0.161 \\
\hline 1170R09 & Core & 55.87 & $<0.05$ & 3.65 & 0.84 & 5.97 & 0.15 & 33.28 & 0.95 & $<0.1$ & 100.8 & 0.909 & 0.133 \\
\hline 1170R21 & & 56.08 & $<0.05$ & 3.37 & 0.87 & 5.83 & 0.14 & 33.46 & 1.19 & $<0.1$ & 101.1 & 0.911 & 0.148 \\
\hline $1170 \mathrm{R} 25$ & Core & 56.38 & 0.03 & 3.13 & 0.81 & 5.71 & 0.16 & 34.02 & 0.83 & $<0.1$ & 101.2 & 0.914 & 0.148 \\
\hline 1170R27 & & 56.20 & $<0.05$ & 3.00 & 0.73 & 5.87 & 0.06 & 33.30 & 1.35 & $<0.1$ & 100.6 & 0.910 & 0.141 \\
\hline 1170R28 & Core & 56.24 & $<0.05$ & 3.35 & 0.81 & 5.72 & 0.16 & 32.62 & 1.14 & $<0.1$ & 100.2 & 0.910 & 0.139 \\
\hline 1176R03 & & 56.99 & $<0.05$ & 2.55 & 0.78 & 5.91 & 0.13 & 33.79 & 1.00 & $<0.1$ & 101.3 & 0.911 & 0.171 \\
\hline 1176R15 & & 56.14 & $<0.05$ & 3.41 & 0.83 & 5.89 & 0.14 & 33.92 & 0.94 & $<0.1$ & 101.4 & 0.911 & 0.140 \\
\hline DR01-106 & Core & 54.49 & 0.06 & 3.86 & 0.99 & 5.84 & 0.11 & 32.66 & 1.35 & 0.10 & 99.5 & 0.909 & 0.146 \\
\hline DR11-003 & & 55.83 & $<0.05$ & 3.42 & 0.79 & 5.75 & 0.13 & 33.05 & 1.57 & 0.12 & 100.7 & 0.911 & 0.134 \\
\hline
\end{tabular}

The $\mathrm{Na}_{2} \mathrm{O}$ and $\mathrm{K}_{2} \mathrm{O}$ contents are lower than detection limit of analyses, $<0.03$ wt. $\%$. STD standard deviation, $\mathrm{N}$ numbers of analyses, $\mathrm{Mg} \# \mathrm{Mg} /(\mathrm{Mg}$ $\left.+\mathrm{Fe}^{\text {total }}\right)$ atomic ratio, $\mathrm{Cr} \# \mathrm{Cr} /(\mathrm{Cr}+\mathrm{Al})$ atomic ratio

Table 14.8 Representative major element compositions of clinopyroxene in peridotites from the Yokoniwa Rise

\begin{tabular}{|c|c|c|c|c|c|c|c|c|c|c|c|c|c|}
\hline Sample & Note & $\mathrm{SiO}_{2}$ & $\mathrm{TiO}_{2}$ & $\mathrm{Al}_{2} \mathrm{O}_{3}$ & $\mathrm{Cr}_{2} \mathrm{O}_{3}$ & $\mathrm{FeO}$ & $\mathrm{MnO}$ & $\mathrm{MgO}$ & $\mathrm{CaO}$ & $\mathrm{Na}_{2} \mathrm{O}$ & Total & $\mathrm{Mg \#}$ & $\mathrm{Cr} \#$ \\
\hline 1170R01 & & 52.28 & 0.07 & 4.48 & 1.42 & 2.35 & $<0.1$ & 16.73 & 23.53 & 0.05 & 101.1 & 0.927 & 0.175 \\
\hline \multirow[t]{4}{*}{ 1170R04 } & Average & 52.86 & 0.22 & 3.03 & 1.17 & 2.42 & $<0.1$ & 17.11 & 22.95 & 0.37 & 100.3 & 0.927 & 0.206 \\
\hline & $\operatorname{STD}(\mathrm{N}=15)$ & 0.78 & 0.07 & 0.39 & 0.21 & 0.10 & $<0.1$ & 0.40 & 0.28 & 0.08 & 0.8 & 0.002 & \\
\hline & Leuco & 53.14 & 0.58 & 2.20 & 0.60 & 2.45 & $<0.1$ & 17.75 & 22.53 & 0.40 & 99.8 & 0.928 & 0.155 \\
\hline & $\operatorname{STD}(\mathrm{N}=3)$ & 0.48 & 0.03 & 0.20 & 0.10 & 0.07 & $<0.1$ & 0.15 & 0.19 & 0.07 & 0.9 & 0.002 & \\
\hline \multirow[t]{2}{*}{ 1170R09 } & Average & 51.92 & 0.09 & 4.55 & 1.39 & 2.42 & $<0.1$ & 16.96 & 22.82 & 0.07 & 100.4 & 0.926 & 0.170 \\
\hline & $\operatorname{STD}(\mathrm{N}=3)$ & 0.25 & 0.01 & 0.24 & 0.04 & 0.16 & $<0.1$ & 0.48 & 1.22 & 0.00 & 0.4 & 0.003 & \\
\hline $1170 \mathrm{R} 25$ & Core & 51.77 & 0.11 & 4.37 & 1.38 & 2.82 & 0.14 & 17.25 & 22.37 & 0.04 & 100.3 & 0.916 & 0.175 \\
\hline 1170R27 & & 52.57 & 0.05 & 3.57 & 0.97 & 2.49 & $<0.1$ & 17.67 & 22.65 & 0.05 & 100.1 & 0.927 & 0.153 \\
\hline $1170 \mathrm{R} 28$ & & 52.25 & 0.11 & 4.29 & 1.22 & 2.52 & 0.12 & 16.56 & 23.80 & 0.08 & 101.0 & 0.921 & 0.160 \\
\hline 1176R03 & & 53.69 & 0.14 & 2.86 & 1.21 & 2.55 & $<0.1$ & 17.19 & 22.52 & 0.34 & 100.6 & 0.923 & 0.222 \\
\hline 1176R09 & & 52.14 & 0.09 & 4.34 & 1.46 & 2.60 & 0.12 & 17.07 & 23.02 & 0.02 & 100.9 & 0.921 & 0.184 \\
\hline 1176R15 & & 52.94 & 0.09 & 4.50 & 1.34 & 2.89 & $<0.1$ & 17.42 & 21.92 & 0.15 & 101.4 & 0.915 & 0.166 \\
\hline 1176R20 & & 53.04 & 0.11 & 4.01 & 1.24 & 2.08 & $<0.1$ & 16.64 & 23.85 & 0.17 & 101.2 & 0.935 & 0.172 \\
\hline DR01-106 & & 52.43 & 0.08 & 3.77 & 0.95 & 2.53 & $<0.1$ & 17.47 & 23.22 & 0.06 & 100.7 & 0.925 & 0.145 \\
\hline DR01-115 & & 52.45 & 1.33 & 2.86 & 1.12 & 2.64 & $<0.1$ & 17.28 & 22.34 & 0.50 & 100.6 & 0.921 & 0.208 \\
\hline DR11-003 & & 52.98 & 0.06 & 4.07 & 1.10 & 2.71 & 0.10 & 17.62 & 22.48 & 0.03 & 101.2 & 0.921 & 0.15 \\
\hline
\end{tabular}

The $\mathrm{K}_{2} \mathrm{O}$ and $\mathrm{NiO}$ contents are lower than detection limit of analyses, $<0.03$ and $<0.1$ wt. $\%$, respectively. STD standard deviation, $N$ numbers of analyses, $\mathrm{Mg} \# \mathrm{Mg} /\left(\mathrm{Mg}+\mathrm{Fe}^{\text {total }}\right)$ atomic ratio, $\mathrm{Cr} \# \mathrm{Cr} /(\mathrm{Cr}+\mathrm{Al})$ atomic ratio

\subsection{Discussions}

\subsubsection{Petrogenesis of Gabbros from the $25^{\circ} \mathrm{S}$ OCC}

In-situ samplings of the mid-ocean ridge gabbros provide us with direct insight into their compositions. These data allow us to compare the results from other ocean floors and to discuss models of how the lower oceanic crusts form.

The petrological characteristics of the gabbros in the $25^{\circ} \mathrm{S}$ OCC are similar to those described from the ultraslowspreading ocean ridges (Dick et al. 2000), where the magmatic activity is low. There are good correlations between plagioclase and clinopyroxene in the studied samples. This is consistent with chemical trends caused by crystallization of plagioclase and clinopyroxene (+olivine) (Fig. 14.6). Differences in the major-element compositions between the oceans can be seen in the diagram. Gabbros from the ultraslow to slow-spreading ocean ridges (MARK area of the Mid-Atlantic ridge and Atlantis Bank of the Southwest Indian Ridge) have lower plagioclase anorthite contents for a given clinopyroxene $\mathrm{Mg} \#$ than those in gabbros from the fast-spreading ocean ridge (Hess Deep and/or Pito Deep of East Pacific) (Perk et al. 2007) (Fig. 14.6). Low-anorthite content of plagioclase at the same $\mathrm{Mg \#}$ of clinopyroxene can be considered as analogues to the parameter $\mathrm{Na}_{8}$ defined by Klein and Langmuir (1987), which is interpreted to be a good indicator of the extent of partial melting. Since a general trend of an increasing extent of partial melting is moved toward higher $\mathrm{Ca}$, a higher anorthite content of plagioclase is expected to be crystallized from hypothetical 
Table 14.9 Representative major element compositions of spinel in peridotites from the Yokoniwa Rise

\begin{tabular}{|c|c|c|c|c|c|c|c|c|c|c|c|c|c|c|}
\hline$\underline{\text { Sample }}$ & Note & $\mathrm{TiO}_{2}$ & $\mathrm{Al}_{2} \mathrm{O}_{3}$ & $\mathrm{Cr}_{2} \mathrm{O}_{3}$ & $\mathrm{FeO}$ & $\mathrm{MnO}$ & $\mathrm{MgO}$ & $\mathrm{NiO}$ & Total & Mg\# & $\mathrm{Cr} \#$ & $\mathrm{YCr}$ & YAl & $\mathrm{YFe}^{3+}$ \\
\hline \multirow[t]{2}{*}{ 1170R01 } & Average & 0.06 & 36.35 & 30.42 & 16.03 & 0.19 & 16.24 & 0.17 & 99.5 & 0.694 & 0.360 & 34.5 & 61.5 & 3.9 \\
\hline & $\operatorname{STD}(\mathrm{N}=8)$ & 0.01 & 0.81 & 0.76 & 0.54 & 0.01 & 0.53 & 0.02 & 0.5 & 0.022 & 0.011 & 0.9 & 1.3 & 0.6 \\
\hline \multirow[t]{2}{*}{ 1170R01 Low-Cr } & Average1 & $<0.05$ & 42.18 & 25.52 & 16.42 & 0.21 & 15.34 & 0.19 & 99.9 & 0.643 & 0.289 & 28.4 & 70.1 & 1 . \\
\hline & $\operatorname{STD}(\mathrm{N}=3)$ & & 0.64 & 0.48 & 1.47 & 0.04 & 1.99 & 0.01 & 0.8 & 0.075 & 0.006 & 0.1 & 1.8 & 1.7 \\
\hline 1170R04 High-Ti & & 0.66 & 22.69 & 42.66 & 22.15 & 0.32 & 11.42 & 0.13 & 100.0 & 0.524 & 0.558 & 53.1 & 42.1 & 4.8 \\
\hline 1170R04 & & 0.48 & 22.01 & 44.16 & 21.61 & 0.31 & 11.90 & 0.12 & 100.6 & 0.545 & 0.574 & 54.5 & 40.5 & 5.0 \\
\hline 1170R04 & & 0.25 & 23.25 & 42.81 & 22.30 & 0.32 & 11.23 & $<0.1$ & 100.3 & 0.516 & 0.553 & 52.7 & 42.7 & 4.6 \\
\hline \multirow[t]{2}{*}{ 1170R09 } & Average & $<0.05$ & 37.95 & 29.74 & 15.33 & 0.20 & 16.27 & 0.17 & 99.7 & 0.690 & 0.345 & 33.5 & 63.7 & 2.8 \\
\hline & $\operatorname{STD}(\mathrm{N}=$ & & 1.07 & 0.68 & 0.59 & 0.02 & 0.47 & 0.02 & 0.8 & 0.013 & 0.011 & 1.0 & 1.2 & 0.4 \\
\hline \multirow[t]{2}{*}{ 1170R09 High-Cr } & Average1 & $<0.05$ & 24.88 & 41.22 & 22.66 & 0.29 & 10.80 & 0.08 & 99.9 & 0.498 & 0.526 & 50.4 & 45.4 & 4.2 \\
\hline & $\operatorname{STD}(\mathrm{N}=2)$ & & 0.95 & 0.79 & 0.20 & 0.01 & 0.09 & 0.01 & 0.0 & 0.002 & 0.014 & 1.2 & 1.5 & 0.3 \\
\hline \multirow[t]{2}{*}{ 1170R21 } & Average & $<0.05$ & 35.56 & 33.51 & 13.91 & 0.20 & 16.82 & 0.17 & 100.2 & 0.715 & 0.387 & 37.8 & 59.8 & \\
\hline & $\operatorname{STD}(\mathrm{N}=3)$ & & 0.19 & 0.18 & 0.18 & 0.02 & 0.12 & 0.02 & 0.3 & 0.003 & 0.000 & 0.1 & 0.1 & 0.1 \\
\hline \multirow[t]{2}{*}{$1170 \mathrm{R} 23$} & Average & 0.05 & 36.33 & 31.78 & 15.65 & 0.20 & 16.28 & 0.17 & 100.5 & 0.690 & 0.370 & 35.8 & 61.0 & 3.2 \\
\hline & $\mathrm{J}=21)$ & 0.01 & 0.74 & 0.40 & 0.34 & 0.02 & 0.28 & 0.02 & 0.9 & 0.011 & 0.006 & 0.5 & 0.8 & 0.4 \\
\hline \multirow[t]{2}{*}{ 1170R25 } & Average & 0.06 & 38.25 & 30.01 & 14.13 & 0.18 & 16.39 & 0.19 & 99.2 & 0.696 & 0.345 & 33.9 & 64.4 & 1. \\
\hline & $\operatorname{STD}(N=7)$ & 0.04 & 0.65 & 0.44 & 0.35 & 0.04 & 0.21 & 0.05 & 0.6 & 0.010 & 0.010 & 0.7 & 0.7 & 0.3 \\
\hline \multirow[t]{2}{*}{ 1170R027 } & Average & 0.05 & 35.64 & 31.96 & 15.75 & 0.21 & 15.89 & 0.15 & 99.7 & 0.682 & 0.376 & 36.4 & 60.5 & 3.0 \\
\hline & $\operatorname{STD}(\mathrm{N}=11)$ & 0.01 & 0.48 & 1.10 & 0.82 & 0.02 & 0.48 & 0.03 & 0.8 & 0.016 & 0.010 & 1.1 & 0.8 & 0 \\
\hline \multirow[t]{2}{*}{ 1170R028 } & Average & 0.07 & 37.30 & 30.70 & 15.25 & 0.21 & 16.45 & 0.17 & 100.2 & 0.696 & 0.356 & 34.5 & 62.6 & 2. \\
\hline & $\mathrm{D}(\mathrm{N}=4)$ & 0.01 & 0.65 & 0.51 & 0.41 & 0.02 & 0.41 & 0.02 & 1.4 & 0.010 & 0.003 & 0.4 & 0.3 & 0. \\
\hline 1176R03 I & & 0.37 & 22.14 & 43.79 & 19.55 & & 12.58 & 0.12 & 98.9 & & 70 & 54.4 & 41.0 & \\
\hline 1176R03 & & 0.25 & 22.38 & 43.52 & 19.46 & 0.29 & 12.63 & 0.09 & 98.6 & 0.586 & 0.566 & 54.0 & 41.4 & 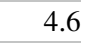 \\
\hline 1176R03 & & 0.11 & 24.50 & 42.12 & 18.97 & 0.32 & 13.47 & 0.09 & 99.6 & 0.613 & 0.536 & 51.0 & 44.2 & 4 \\
\hline \multirow[t]{2}{*}{ 1176R09 } & Average & $<0.05$ & 18.18 & 16.33 & 8.50 & 0.12 & 7.98 & 0.09 & 51.2 & & 0.376 & 36.3 & 60.3 & \\
\hline & $\mathrm{STD}(\mathrm{N}=$ & & 18.52 & 16.65 & 8.46 & 0.10 & 7.85 & 0.08 & 51.5 & 0.337 & 0.199 & 19.3 & 31.3 & \\
\hline $1176 \mathrm{R} 15 \mathrm{H}$ & & 1.33 & 19.35 & 41.42 & 24.54 & 0.33 & 11.39 & 0.18 & 98.6 & 0.534 & 0.589 & 53.5 & 37.2 & 9. \\
\hline 1176R15 & & 0.66 & 26.41 & 36.22 & 20.78 & 0.25 & 13.96 & 0.15 & 98.4 & 0.630 & 0.479 & 44.1 & 47.9 & 8 \\
\hline 1176R15 & & 0.08 & 35.26 & 29.43 & 17.06 & 0.22 & 16.03 & 0.21 & 98.3 & 0.695 & 0.359 & 33.9 & 60.6 & \\
\hline \multirow[t]{2}{*}{ 1176R20 } & Avera & 0.05 & 36.05 & 30.61 & 15.19 & 0.20 & 16.50 & 0.16 & 98.8 & 0.709 & 0.363 & 34.9 & 61.3 & 3. \\
\hline & $\operatorname{STD}(\mathrm{N}=3)$ & 0.01 & 0.43 & 0.26 & 0.11 & 0.01 & 0.09 & 0.01 & 0.6 & 0.002 & 0.002 & 0.1 & 0.3 & 0. \\
\hline \multirow[t]{2}{*}{ 1176R22 } & Average & $<0.05$ & 18.33 & 15.84 & 7.92 & 0.10 & 8.15 & 0.08 & 50.5 & 0.688 & 0.367 & 35.5 & 61.3 & 3. \\
\hline & STD $(\mathrm{N}=$ & & 17.81 & 15.19 & 7.56 & 0.09 & 8.21 & 0.08 & 49.1 & 0.353 & 0.181 & 17.4 & 30.5 & 1. \\
\hline \multirow[t]{2}{*}{ DR01-106 } & Average & 0.08 & 36.25 & 31.04 & 14.22 & 0.18 & 17.30 & 0.22 & 99.3 & 0.736 & 0.365 & 35.1 & 61.1 & 3. \\
\hline & $\operatorname{STD}(\mathrm{N}=3)$ & 0.01 & 0.29 & 0.20 & 0.20 & 0.01 & 0.05 & 0.04 & 0.1 & 0.003 & 0.003 & 0.2 & 0.5 & 0. \\
\hline \multirow[t]{2}{*}{ DR01-106 Low-Cr } & Average1 & $<0.05$ & 39.01 & 29.28 & 15.18 & 0.18 & 16.69 & 0.18 & 100.6 & 0.699 & 0.335 & 32.6 & 64.7 & 2 \\
\hline & $\operatorname{STD}(\mathrm{N}=3)$ & & 0.39 & 0.21 & 0.45 & 0.02 & 0.30 & 0.02 & 0.5 & 0.011 & 0.004 & 0.4 & 0.3 & 0. \\
\hline DR01-103 High-Ti & & 0.23 & 27.40 & 41.52 & 15.33 & 0.24 & 15.25 & $<0.1$ & 100.1 & 0.674 & 0.504 & 49.0 & 48.3 & 2.7 \\
\hline DR01-103 & & 0.17 & 30.85 & 38.07 & 14.70 & 0.22 & 16.21 & 0.12 & 100.3 & 0.701 & 0.453 & 44.0 & 53.1 & 2. \\
\hline DR01-103 & & 0.10 & 33.97 & 34.85 & 13.78 & 0.18 & 16.97 & 0.16 & 100.0 & 0.725 & 0.408 & 39.6 & 57.6 & 2 \\
\hline DR01-115 High-Ti & & 0.64 & 29.64 & 37.62 & 16.11 & 0.25 & 15.66 & 0.18 & 100.1 & 0.681 & 0.460 & 44.3 & 52.0 & 3. \\
\hline DR01-115 & & 0.54 & 31.11 & 35.95 & 15.93 & 0.19 & 15.73 & 0.19 & 99.7 & 0.683 & 0.437 & 42.1 & 54.3 & 3. \\
\hline DR01-115 & & 0.25 & 22.61 & 46.54 & 17.30 & 0.31 & 13.75 & 0.12 & 100.9 & 0.621 & 0.580 & 56.3 & 40.7 & 3.0 \\
\hline \multirow[t]{2}{*}{ DR09-19 } & Average & 0.08 & 26.87 & 40.83 & 17.84 & 0.24 & 14.66 & 0.13 & 100.7 & 0.650 & 0.505 & 48.1 & 47.2 & 4. \\
\hline & $\operatorname{STD}(\mathrm{N}=6)$ & 0.02 & 0.16 & 0.23 & 0.32 & 0.02 & 0.10 & 0.01 & 0.5 & 0.002 & 0.002 & 0.3 & 0.2 & 0. \\
\hline \multirow[t]{2}{*}{ DR11-003 } & Average & 0.05 & 38.30 & 29.51 & 15.50 & 0.20 & 16.47 & 0.17 & 100.2 & 0.694 & 0.341 & 33.0 & 63.9 & 3.1 \\
\hline & $\operatorname{STD}(N=6)$ & 0.02 & 1.21 & 0.50 & 0.84 & 0.02 & 0.54 & 0.02 & 0.6 & 0.016 & 0.011 & 0.9 & 1.3 & 0 . \\
\hline DR11-008 & Average & 0.06 & 37.08 & 30.63 & 14.53 & 0.18 & 17.06 & 0.19 & 101.5 & 0.722 & 0.357 & 34.5 & 62.2 & 3. \\
\hline
\end{tabular}

The $\mathrm{SiO}_{2}, \mathrm{CaO}, \mathrm{Na}_{2} \mathrm{O}$ and $\mathrm{K}_{2} \mathrm{O}$ are lower than detection limit of analyses, $<0.03$ wt. $\% . M g \# \mathrm{Mg} /\left(\mathrm{Mg}+\mathrm{Fe}^{2+}\right)$ atomic ratio $\left(\mathrm{Fe}^{2+}\right.$ in spinel was calculated from spinel stoichiometry), $C r \# \mathrm{Cr} /(\mathrm{Cr}+\mathrm{Al})$ atomic ratio, $Y C r \mathrm{Cr} /\left(\mathrm{Cr}+\mathrm{Al}+\mathrm{Fe}^{3+}\right)$ atomic ratio, $Y A l \mathrm{Al} /\left(\mathrm{Cr}+\mathrm{Al}+\mathrm{Fe}{ }^{3+}\right)$ atomic ratio, $Y F e^{3+}$ $\mathrm{Fe}^{3+} /\left(\mathrm{Cr}+\mathrm{Al}+\mathrm{Fe}^{3+}\right)$ atomic ratio 
Fig. 14.6 Variations in the major-element compositions of minerals in gabbros from $25^{\circ} \mathrm{S}$. (a) Relationship between the $\mathrm{Mg \#}$ and the $\mathrm{TiO}_{2}$ wt. $\%$ in clinopyroxene. (b) Relationship between the anorthite content in plagioclase and the $\mathrm{Mg \#}$ in clinopyroxene. Tie line between data indicates the same sample. $O l$ olivine-bearing part, $P x$ olivine-free and amphibole-poor part, Amph amphibole-rich part.

The compositional ranges of main cluster of mid-ocean ridge gabbros (Hess Deep and Pito Deep, MARK = Kane fracture zone of the Mid-Atlantic Ridge, Southwestern Indian Ridge), compiled by Coogan et al. (2007), were also shown.
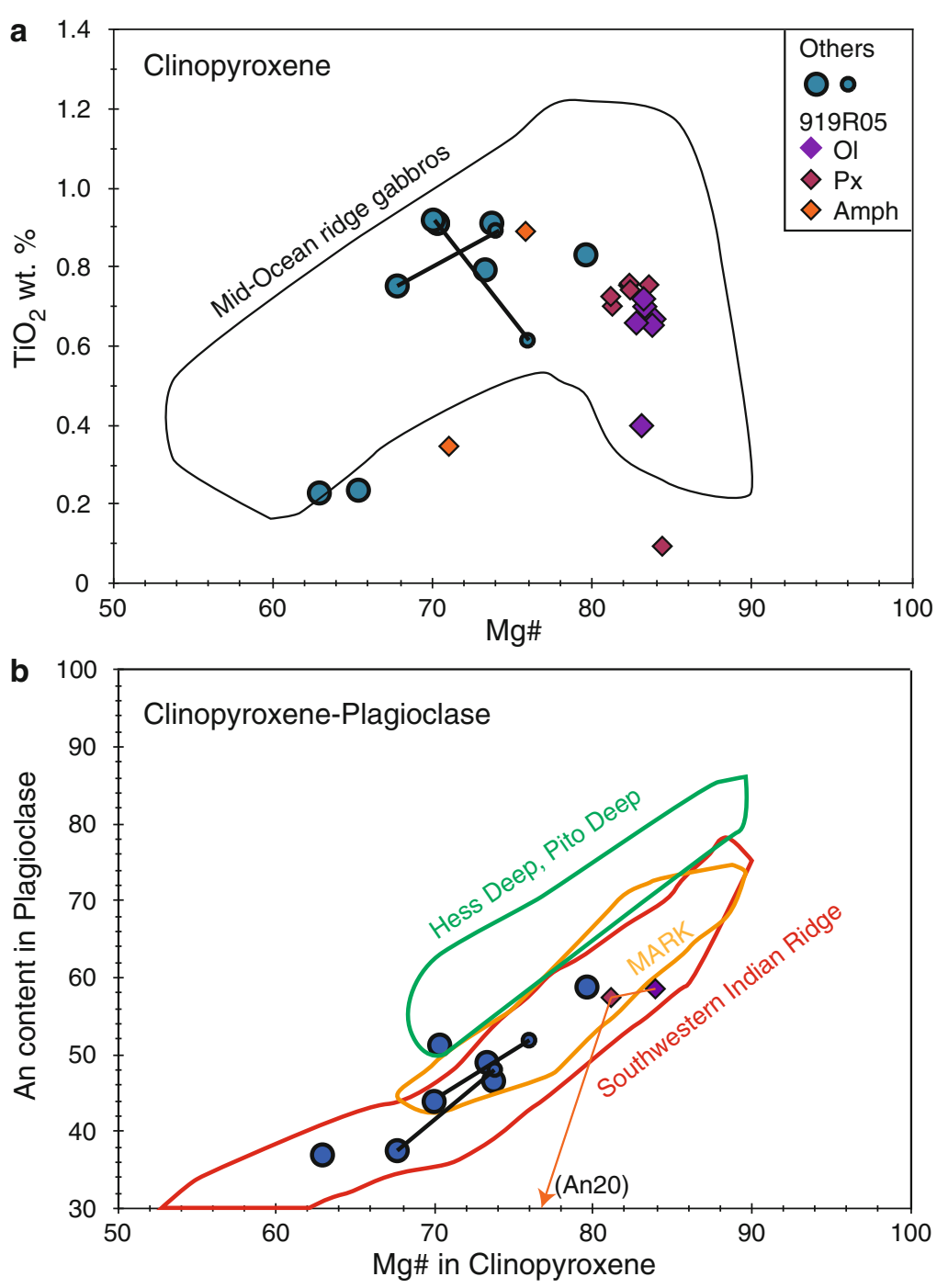

primitive MORB liquids formed at the fast-spreading ridges than at the slow-spreading ridges. Their Mg\#s of mafic minerals in the $25^{\circ} \mathrm{S}$ OCC are, however, too low to be in equilibrium with mantle compositions. This has been long recognized as a "missing primary cumulate" in the slowspreading ridges (Dick et al. 2000). On the other hand, oxide gabbros, rich in both ilmenite and magnetite, and their close association with oxide-poor gabbros, are common features at slow-spreading ridges (Dick et al. 2000). Pigeonite-bearing gabbros were also reported from the upper part of the Hole 735B gabbro of the Southwest Indian Ridge (Ozawa et al. 1991). Since the $25^{\circ} \mathrm{S}$ OCC is located at the segment boundary between CIR-S1 and CIR-S2, fractionated melt compositions can be explained by lateral intrusion of differentiate melt from focused melt flow at the mid-point of the ridge segments. In conclusion, the petrological characteristics of the gabbros in the $25^{\circ} \mathrm{S}$ OCC were formed while the magmatic activity was low, just as those described from the ultraslow-spreading ocean ridges (Dick et al. 2000).

\subsubsection{Effect of Late Modification Due to Infiltration of Multiple Melts on Perdidotites}

It is expected that peridotites associated with leucocratic veins were petrologically and geochemically modified during the formation of the leucocratic veins (e.g., Morishita et al. 2009). Leucocratic veins are observed as networks in several peridotites from the $25^{\circ} \mathrm{S}$ OCC and Yokoniwa Rise. Altered plagioclases, now replaced by fine-grained mineral aggregates (i.e., saussurite), are observed around spinels or as aggregates locally in some peridotites. Samples around the leucocratic veins contain altered plagioclase in spite of the low abundance of clinopyroxenes. Leucocratic veinbearing peridotites show heterogeneity in minerals within single samples, whereas vein-free samples are basically homogeneous in minerals.

Petrological and geochemical features indicate that plagioclases ( \pm clinopyroxenes) in the studied peridotites 


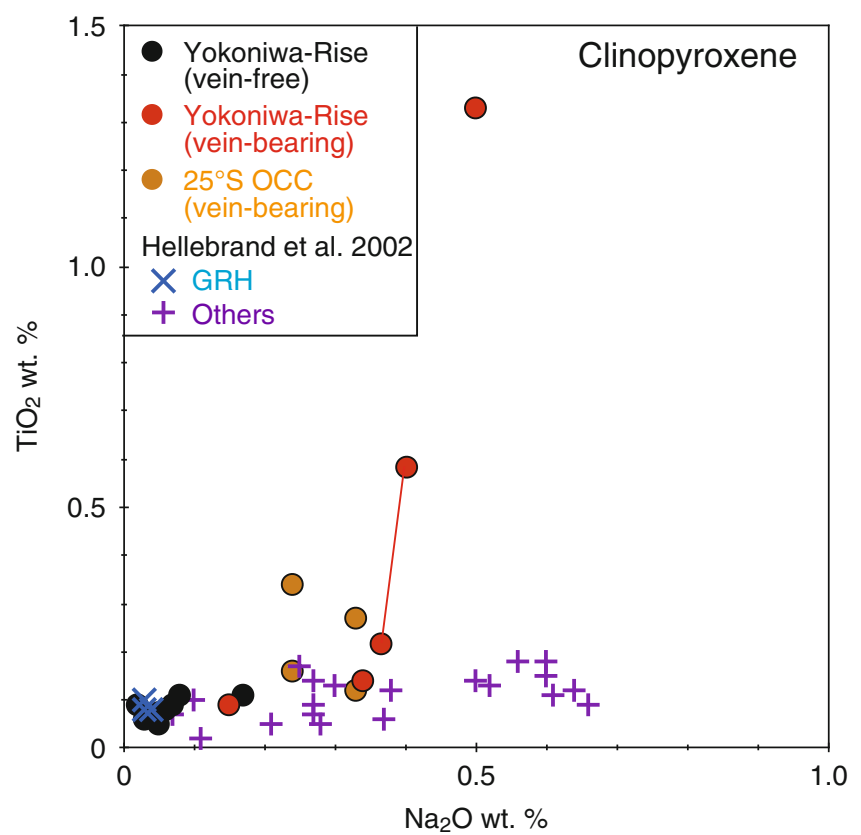

Fig. 14.7 Compositional relationships between $\mathrm{TiO}_{2}$ wt. $\%$ and $\mathrm{Na}_{2} \mathrm{O}$ wt.\% in clinopyroxene in peridotite samples recovered from the $25^{\circ} \mathrm{S}$ OCC and Yokoniwa Rise. Tie line between data indicates the same sample. $C I R$ Central Indian Ridge, GRH Green Rock Hill

both in the $25^{\circ} \mathrm{S}$ OCC and the Yokoniwa Rise were of magmatic origin related to the formation of leucocratic veins at olivine-plagioclase stability conditions $(<0.8 \mathrm{GPa})$ rather than the metamorphic origin of the subsolidus reaction from pyroxenes and spinel during the exhumation of upper mantle materials from beneath the ocean floor. During the formation of plagioclase, the alumina in the spinels in the peridotites was partly consumed to form plagioclase, and incompatible elements, such as $\mathrm{TiO}_{2}$ and $\mathrm{Na}_{2} \mathrm{O}$ would be added to the precursor minerals (e.g., clinopyroxene) (Figs. 14.7 and 14.8). Clinopyroxenes in the samples with leucocratic veins are, therefore, distinctively higher in both $\mathrm{Na}_{2} \mathrm{O}$ and $\mathrm{TiO}_{2}$ contents (Fig. 14.7) than those in the veinfree samples. The $\mathrm{Cr} \#$ and $\mathrm{TiO}_{2}$ contents of the spinels in the peridotites with leucocratic veins show a wide range of variation, $0.3-0.6$ for $\mathrm{Cr} \#$ and up to $1.3 \mathrm{wt} . \%$ for $\mathrm{TiO}_{2}$ (Fig. 14.8). Low $\mathrm{Cr} \#$ and $\mathrm{TiO}_{2}$ of the chemical range of spinels from the Yokoniwa Rise are, on the other hand, the same as those from the vein-free samples. Spinels far from the leucocratic vein might, therefore, retain their original geochemical signatures before they were modified by interaction with the melts responsible for the formation of leucocratic vein (Fig. 14.8).

The melts which infiltrated into the peridotite samples had different chemical compositions from less evolved to highly evolved signatures. Although primary plagioclase is not observed in the leucocratic veins, secondary minerals such as chlorite and tremolitic/actinolitic amphibole

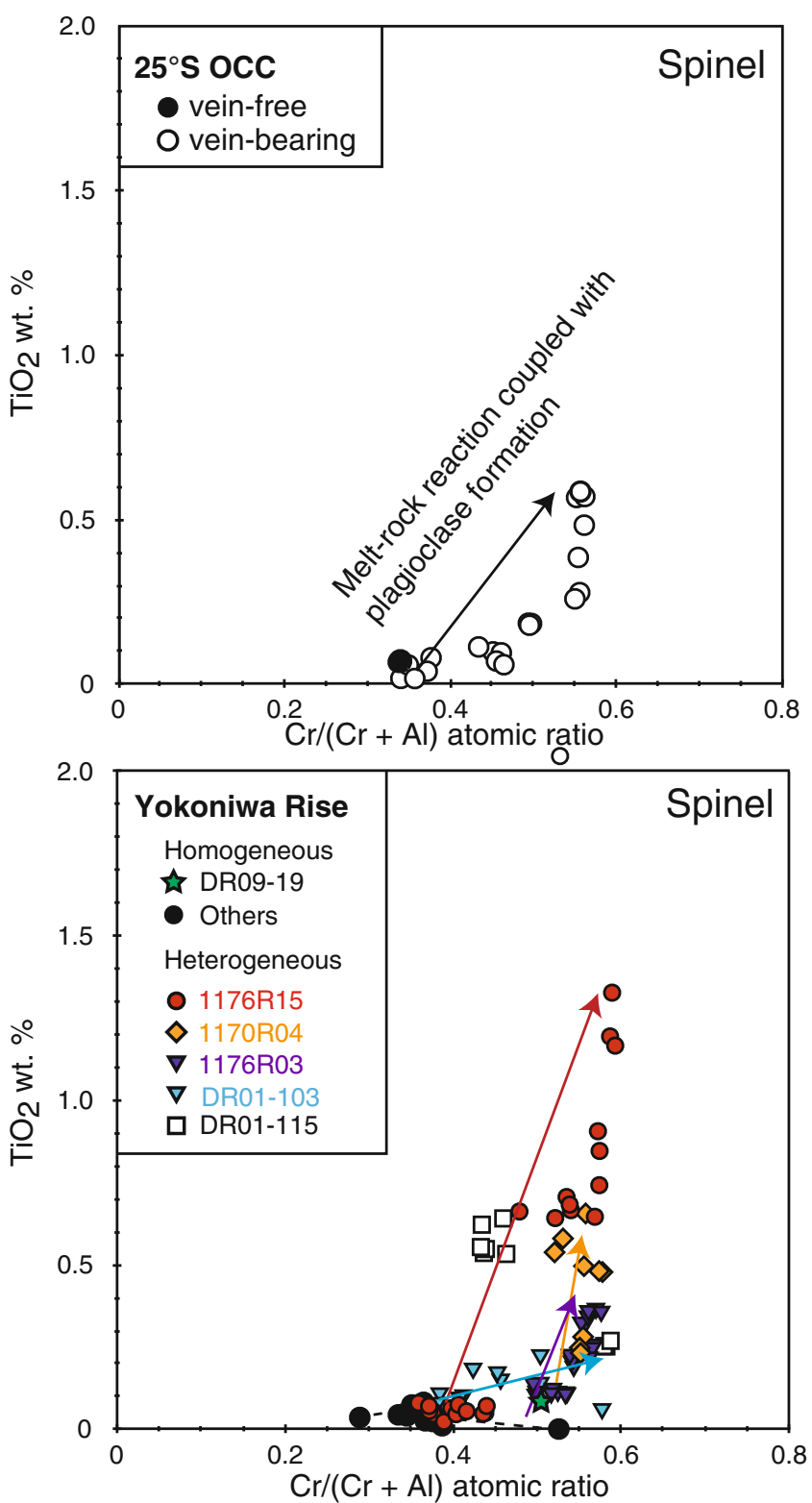

Fig. 14.8 Compositional relationships between $\mathrm{Cr} /(\mathrm{Cr}+\mathrm{Al}$ ) (atomic ratio) and $\mathrm{TiO}_{2}$ wt. $\%$ in spinels from $25^{\circ} \mathrm{S}$ OCC (upper panel) (data are from Morishita et al. (2009)) and Yokoniwa Rise (lower panel)

indicates their original plagioclase-bearing litholoties. Altered plagioclases coupled with the presence of clinopyroxene and brown amphibole in the leucocratic veins suggest their origins from plagioclase-bearing mafic rocks, i.e., gabbroic rocks. On the other hand, zircons in some leucocratic veins indicate highly evolved signatures, such as granitic compositions (Morishita et al. 2009). The variations in the infiltrated melts are also supported by the variations in the maximum $\mathrm{TiO}_{2}$ contents in minerals along the leucocratic veins in peridotites (e.g., $0.4-1.3 \mathrm{wt} . \%$ for spinels with the Cr\# around 0.5). During the infiltration of a small amount of melt, the degree of chemical modifications 
depends on the combination of an effective melt/rock ratio, melt compositions and $\mathrm{P}-\mathrm{T}$ conditions on reactions. The size of the leucoratic veins is usually less than $1 \mathrm{~cm}$ in thickness. No systematic variations between the size of the leucocratic veins and modified mineral compositions are observed in the studied peridotites. The differences in the $\mathrm{TiO}_{2}$ contents among those modified minerals are interpreted to be caused by the differences in melt compositions from less evolved to highly evolved compositions rather than the differences in effective melt/rock ratios. The $\mathrm{TiO}_{2}$ content of melts are generally changed during the fractional crystallization as shown in the chemical variations of clinopyroxene in the $25^{\circ} \mathrm{S}$ OCC gabbros. Highly evolved gabbroic rocks were reported from both the slow-spreading ridges (Bloomer et al. 1989; Mével et al. 1991; Cannat et al. 1992; Morishita et al. 2004) and the fast-spreading ridges (Constantin 1999). Physical conditions under slow-spreading ridges are characterized by a highly attenuated magma supply and a high rock/melt ratio during melt ascending. We favor the theory that the differences in chemical compositions of the infiltrated melts in the peridotites were formed as an in-situ fractionation from a MORB-type melt during the ascent in the mantle while the magmatic activity was low in the intermediate-spreading mid-ocean ridges.

\subsubsection{The Primary Features of Peridotites: Implications for the Formation of Serpentine-Dominated Seafloor}

The primary features of peridotites recovered from the studied area suggest their relatively depleted signatures in melt components. Leucocratic vein-free samples are basically homogeneous in mineral chemistry at each sample scale. The majority of spinel compositions in the Yokoniwa Rise peridotites is around 0.35 for $\mathrm{Cr} \#$ and $<0.1 \mathrm{wt} \%$ for $\mathrm{TiO}_{2}$ contents (Fig. 14.8). It should be emphasized that one sample (DR09-19) is distinctively high in Cr\# (Fig. 14.8). Low$\mathrm{TiO}_{2}$ of mineral compositions in the leucocratic vein-bearing samples are less affected by the chemical modifications, i.e., primary features of peridotite prior to the chemical modifications from the infiltrated melts. Primary spinel compositions of 1176R05 and DR01-103 are around 0.35 for $\mathrm{Cr} \#$, whereas those of 1170R04 and 1176R03 are likely to be higher in $\mathrm{Cr} \#$ than other samples, around 0.5 (Fig. 14.8). Spinels from the $25^{\circ} \mathrm{S}$ OCC peridotites, leucocratic vein-free and those far from the leucocratic vein-bearing, are also around 0.35 for $\mathrm{Cr} \#$ and $<0.1$ wt. \% for the $\mathrm{TiO}_{2}$ content (Fig. 14.8). The composition of the Green Rock Hills is similar to that of the studied samples. The compositional range of these spinels and $\mathrm{Al}_{2} \mathrm{O}_{3}$ content of orthopyroxene porphyroclasts are overlapped with higher and lower ranges of plagioclase-free CIR abyssal peridotites
(Hellebrand et al. 2002), respectively (Fig. 14.9). According to these results, the granular peridotite samples collected from the studied area are residue left after moderate to high degrees of partial melting (13-15\% in the majority and up to $17 \%$ based on the method of Hellebrand et al. (2001)). It should be realized that this study, coupled with previous results, confirms a wide exposure of relatively depleted peridotites on the ocean floor in the studied region.

Niu and Hékinian (1997) reported a correlation between ridge spreading rate and the degree of partial melting in abyssal peridotites, i.e., the idea that the extent of mantle melting beneath normal ocean ridges increases with increasing spreading rate. The studied peridotites are plotted between those from fast-spreading ridges and slow-spreading ridges except for hotspot related areas (Fig. 14.9). In this context, the moderate to high degree of partial melting of the studied peridotites appears to be consistent with those expected for an "intermediate"spreading ridge. In particular, the higher compositional range of the Cr\# of spinels in peridotites recovered from the southern end of the CIR is also likely consistent with the increase of the spreading rate toward the south along the CIR. Moderately to highly depleted melt components in the studied peridotites can be explained as being residue after a relatively high-melt productivity period in intermediate-spreading ridges.

We also discuss several models for the origin of depleted peridotites in the studied area because peridotites with depleted signatures in melt components were recently reported from oceanic core complexes where fertile peridotites are expected to be recovered (Seyler et al. 2007; Tamura et al. 2008; Dick et al. 2010) (Fig. 14.9). Depleted peridotites were also already recovered from other ultraslow to slow-spreading ridges without a hot spot effect (Bonatti et al. 1992, 2003; Seyler et al. 2003; Brunelli et al. 2006; Morishita et al. 2007; Godard et al. 2008). These studies indicate that large variations in the melting degree of midocean ridge peridotites are probably not simply related to the spreading rate. Several models can be applied for the occurrence of the depleted peridotites even where the degree of partial melting is expected to be low: (i) relics of older partial melting (Seyler et al. 2003, 2007; Liu et al. 2008), (ii) variations of equilibration temperatures probably related to variations of advection velocity below the ridge (Bonatti et al. 2003), and (iii) residue related to the latest melting events, i.e., the present mid-ocean ridge spreading (Morishita et al. 2007). We cannot examine the second case (ii) based on our samples because further samplings in space and time along the CIR are required.

In the last case (iii), we assume that the depleted rocks appear to be very locally formed along main melt conduits. Morishita et al. (2007) described petrological characteristics of a dunite from the ultraslow-spreading Southwest Indian Ridge where fertile clinopyroxene-rich lherzolite is dominant lithology, and reported a relatively depleted sample 


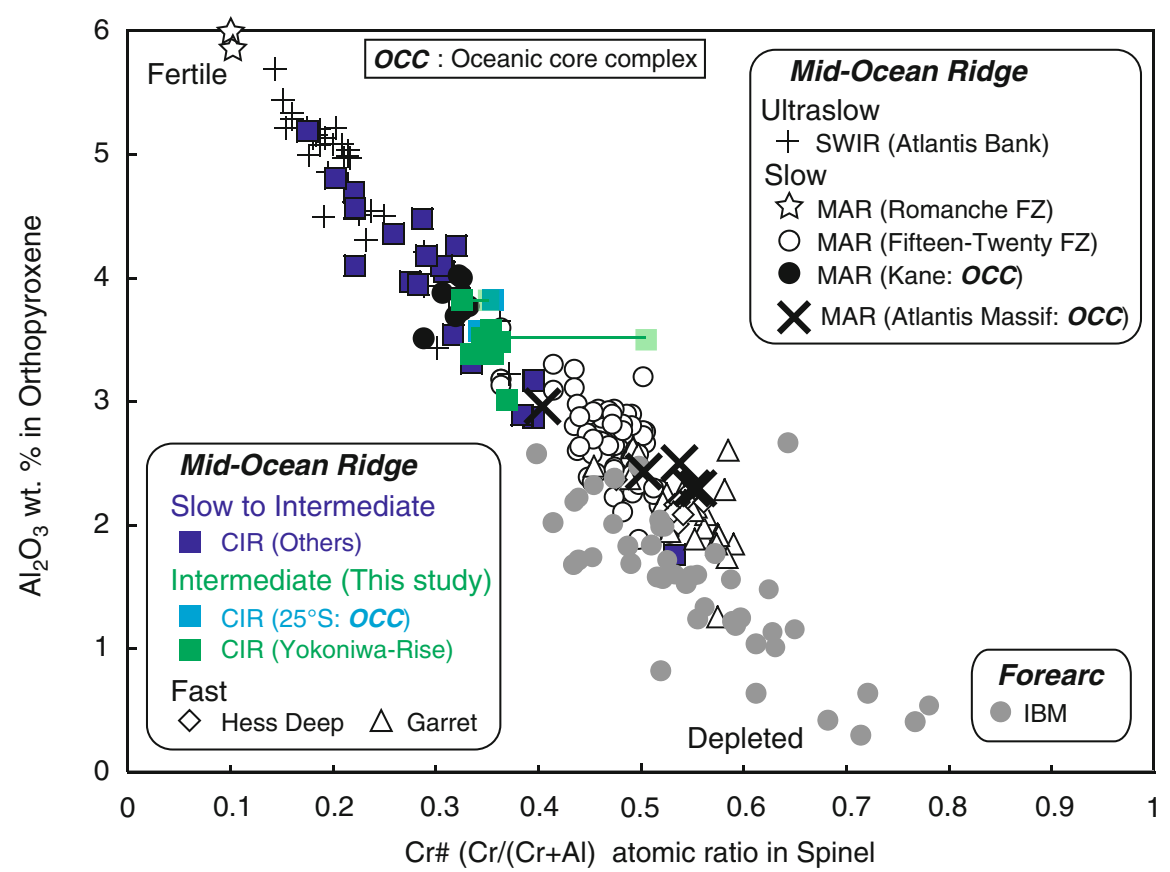

Fig. 14.9 Correlations between $\mathrm{Al}_{2} \mathrm{O}_{3}$ content (wt.\%) in orthopyroxene porphyroclasts and $\mathrm{Cr} \#$ in spinels. Data of the Southwest Indian Ridge (SWIR) are from Atlantis II Fracture Zone of Dick (Dick 1989) and Seyler et al. (2003). The Central Indian Ridge (CIR) are from Hellebrand et al. (2002), Morishita et al. (2009). The MidAtlantic Ridge (MAR) are as follows: Atlantis Massif (Oceanic core complex: OCC) are from Seyler et al. (2007) and Tamura et al. (2008),
Romanche fracture zone (FZ) are from Bonatti et al. (1993), FifteenTwenty FZ are from Godard et al. (2008), and Kane megamullion (oceanic core complex) are from Dick et al. (2010). The Garret and Hess Deep of East Pacific Rise (EPR) are from Constantin (1999) and Dick and Natland (Dick and Natland 1996), respectively. The IzuBonin-Mariana (IBM) forearc are from Parkinson and Pearce (1998) and Zanetti et al. (2006)

melting of relatively fertile peridotite beneath the ancient depleted domain might be infiltrated into the upwelled ancient depleted domain, resulting in the formation of gabbroic networks/veins in peridotites. Continuous dragging of the oceanic plate causes fracturing of the uppermost section of the oceanic plate along the ocean ridge. Seawater would have penetrated along the cracks and reacted with peridotites to form serpentinites. Because serpetinized peridotites are less dense than the surrounding host peridotites, serpentinized peridotites can be locally exposed on the seafloor. Further studies on the melting ages of peridotites, such as Os-isotopiec dating, are, however, required to make clear whether or not ancient melting regions are the origin for the studied peridotites.

The ancient melting model (i) can be favored over other model as an explanation for a wide exposure of serpentinized peridotite in the studied area. In this case, depleted peridotites formed as residue, after an ancient partial melting event or evenets, should be survived and would be incorporated beneath a mid-ocean ridge center without significant modifications. Less or no partial melting is expected in the ancient depleted peridotite domain during the upwelling beneath the spreading ridges, resulting in a very limited amount of igneous crust formations at the spreading center. Melts caused by the following partial

\subsection{Conclusions}

We succeeded three submersible expeditions of the SHINKAI 6500 and one dredge expedition of the Hakuhomaru in the south end of the intermediate-spreading Central Indian Ridge, where the Kairei hydrothermal filed is located. Peridotites and their-related gabbroic rocks were widely recovered from the studied region. Here we summarize petrological and mineralogical characteristics the peridottes 
and related gabbros from the oceanic core complex at a latitude of $25^{\circ}$ South $\left(25^{\circ} \mathrm{S}\right.$ OCC) and the Yokoniwa Rise around the Kairei hydrothermal field.

Gabbros from the $25^{\circ} \mathrm{S}$ OCC are olivine gabbro, gabbro, gabbronorite (granular orthopyroxene-bearing gabbro) and oxide gabbro. The petrological characteristics of the gabbros are similar to those described from the ultraslow-spreading ocean ridges. The gabbros in the $25^{\circ} \mathrm{S}$ OCC were probably formed while the magmatic activity was low.

The peridotites are classified into two types: a granular peridotite and a highly deformed type of rock. The latter was recovered from the top surface of the $25^{\circ} \mathrm{S}$ OCC. Petrological characteristics of granular peridotites (clinopyroxene-bearing harzburgite to lherzolite) are basically the same between the $25^{\circ} \mathrm{S}$ OCC and the Yokoniwa Rise. Several samples were intruded by leucocratic veins. Plagioclase, now completely altered, was observed in several samples with thick leucocratic veins. The primary features of peridotites are residue left after moderate to high degrees of partial melting (13-15\% in the majority and up to $17 \%$ ). The partial melting of these peridotites is followed by chemical modification through interaction with a wide range of melts from relatively less evolved to highly evolved characteristics. The differences in chemical compositions of the infiltrated melts in the peridotites were probably formed as an in-situ fractionation from a MORBtype melt during the ascent in the mantle. Two models for the origin of depleted peridotites in the studied area: (1) residue related to the latest melting events of the present mid-ocean ridge spreading, and (2) residue related to an ancient partial melting event or evenets. In the case (1), the studied peridotites are residue after a relatively high-melt productivity period in intermediate-spreading ridges. In the case (2), the studied peridotites are a geochemically distinctive domain that has suffered from partial melting in the past.

\begin{abstract}
Acknowledgements We are grateful to captains, crews and the scientific parties of the Yokosuka, the SHINKAI team, the R/V Hakuhomaru who contributed to the success of the cruises. This study was partly supported by a Grants-in-Aid for Scientific Research from the Ministry of Education, Culture, Sports, Science and Technology of Japan (MEXT) on Innovative Areas "Project TAIGA: Trans-crustal Advection and In-situ biogeochemical process of Global sub-seafloor Aquifer" led by Prof. Urabe and a Grants-in-Aid for Scientific Research of the MEXT (No. 17740349) to T.M. We also deeply thanks to Shoji Arai, Susumu Umino, Nobu Seama, Tomoyuki Mizukami, Akihiro Tamura and Sumiaki Machi for their discussions. The paper was greatly improved by reviews by two anonymous reviewers. Daniel Gauss improved English in the manuscript.
\end{abstract}

Open Access This chapter is distributed under the terms of the Creative Commons Attribution Noncommercial License, which permits any noncommercial use, distribution, and reproduction in any medium, provided the original author(s) and source are credited.

\section{References}

Bloomer SH, Natland JH, Fisher RL (1989) Mineral relationships in gabbroic rocks from fracture zones of Indian Ocean ridges: evidence for extensive fractionation, parental diversity and boundary-layer recrystallization. Geol Soc Spec Pub 42:107-124. doi:10.1144/GSL.SP.1989.042.01.07

Bonatti E, Peyve A, Kepezhinskas P, Kurentsova N, Seyler M, Skolotnev S, Udintsev G (1992) Upper mantle heterogeneity below the Mid-Atlantic Ridge, $0^{\circ}-15^{\circ}$ N. J Geophys Res 97 (B4):4461-4476

Bonatti E, Seyler M, Sushevskaya N (1993) A cold suboceanic mantle belt at the Earth's Eauator. Science 262(5119):315-320. doi: $10.2307 / 2881562$

Bonatti E, Ligi MD, Brunelli A, Cipriani P, Fabretti V, Ferrante L, Gasperini L, Ottolini L (2003) Mid-Atlantic Ridge and temporal variations in the formation of oceanic lithosphere. Nature 423 (6939):499-505. doi:10.1038/nature01594

Briais A (1995) Structural-analysis of the segmentation of the CentralIndian-Ridge between 20-degrees-30's and 25-degrees-30's (Rodriguez-Triple-Junction). Mar Geophys Res 17(5):431-467. doi:10.1111/j.1365-246X.2009.04491.x

Brunelli D, Seyler M, Cipriani A, Ottolini L, Bonatti E (2006) Discontinuous melt extraction and weak refertilization of mantle peridotites at the Vema lithosphereic section (Mid-Atlantic Ridge). J Petrol 47(4):745-771. doi:10.1093/petrology/egi092

Cannat M, Bideau D, Bougault D (1992) Serpentinized peridotites and gabbros in the Mid-Atlantic Ridge axial valley at $15^{\circ} 37^{\prime}$ and $16^{\circ} 52^{\prime} \mathrm{N}$. Earth Planet Sci Lett 109(1-2):87-106. doi:10.1016/ 0012-821X(92)90076-8

Constantin M (1999) Gabbroic intrusions and magmatic metasomatism in harzburgites from the Garret transform fault: implications for the nature of the mantle-crust transition at fast-spreading ridges. Contrib Mineral Petrol 136(1-2):111-130. doi:10.1007/ s004100050527

Coogan LA, Jenkin GRT, Wilson RN (2007) Contrasting cooling rates in the lower oceanic crust at fast- and slow-spreading ridges revealed by geospeedometry. J Petrol 48(11):2211-2231. doi;10. 1093/petrology/egm057

DeMets C, Gordon RG, Argus DF (2010) Geologically current plate motions. Geophys J Int 181(1):1-80. doi:10.1111/j.1365-246X. 2009.04491.x

Dick HJB (1989) Abyssal peridoties, very slow spreading ridges and ocean ridge magmatism. In: Saunders AD, Norry MJ (eds) Magmatism of Ocean Basins. Geological Society Special Publication, Oxford: Blackwells for the Geological Society, no. 42, pp 71-105

Dick HJB, Natland JH(1996) Late-stage melt evolution and transport in the shallow mantle beneath the East pacific Rise. Proc Ocean Drill Prog 147:103-134

Dick HJB, Natland JH, Alt JC, Bach W, Bideau D, Gee JS, Haggas S, Hertogen JGH, Hirth G, Holm PM, Ildefonse B, Iturrino GJ, John BE, Kelley DS, Kikawa E, Kingdon A, LeRoux J, Maeda PJ, Meyer J, Miller PS, Naslund DJ, Niu HR, Robinson Y-L, Snow PT, Stephen RA, Trimby PW, Worm H-U, Yoshinobu A (2000) A long in situ section of the lower ocean crust: results of ODP Leg 176 drilling at the Southwest Indian Ridge. Earth Planet Sci Lett 179(1):31-51

Dick HJB, Lissenberg CJ, Warren JM (2010) Mantle melting, melt transport, and deliver beneath a slow-spreading ridge: the paleoMAR from $23^{\circ} 15^{\prime} \mathrm{N}$ to $23^{\circ} 45^{\prime} \mathrm{N}$. J Petrol 51(1-2):425-467

Gallant RM, Von Damm KL (2006) Geochemical controls on hydrothermal fluids from the Kairei and Edmond Vent Fields, $23^{\circ}-25^{\circ} \mathrm{S}$, 
Central Indian Ridge. Geochem Geophys Geosyst 7(6):1-24. doi:10.1029/2005GC001067

Gamo T, Chiba H, Yamanaka T, Okudaira T, Hashimoto J, Tsuchida S, Ishibashi J, Kataoka S, Tsunogai U, Okamura K, Sano Y, Shinjo R (2001) Chemical characteristics of newly discovered black smoker fluids and associated hydrothermal plumes at the Rodriguez Triple Junction, Central Indian Ridge. Earth Planet Sci Lett 193 (3-4):371-379

Godard M, Lagabrielle Y, Alard O, Harvey J (2008) Geochemistry of the highly depleted peridotites drilled at ODP Sites 127 and 1274 (Fifteen-Twenty Fracture Zone, Mid-Atlantic Ridge): implications for mantle dynamics beneath a slow spreading ridge. Earth Planet Sci Lett 267(3-4):410-425

Hashimoto J, Ohta S, Gamo T, Chiba H, Yamaguchi S, Tsuchida S, Okudaira T, Watabe H, Yamanaka T, Kitazawa M (2001) First hydrothermal vent communities from the Indian Ocean discovered. Zoolog Sci 18(5):717-721

Hellebrand E, Snow JE, Dick HJB, Hofmann AW (2001) Coupled major and trace elements as indicators of the extent of melting in mid-ocean-ridge peridotites. Nature 410:677-681

Hellebrand E, Snow JE, Hoppe P, Hofmann AW (2002) Garnet-field melting and late-stage refertilization in 'residual' abyssal peridotites from the Central Indian Ridge. J Petrol 43 (12):2305-2338

Honsho C, Tamaki K, Fujimoto H (1996) Three-dimensional magnetic and gravity studies of the Rodriguez Triple Junction in the Indian Ocean. J Geophys Res 101(B7):15837-15848

Klein EM, Langmuir CH (1987) Global correlations of ocean ridge basalt chemistry. J Geophys Res 92(B8):8089-8115

Kumagai H, Nakamura K, Toki T, Morishita T, Okino K, Ishibashi J-I, Tsunogai U, Kawaguchi S, Gamo T, Shibuya T, Sawaguchi T, Neo N, Joshima M, Sato T, Takai K (2008) Geological background of the Kairei and Edmond hydrothermal fields along the Central Indian Ridge: implications of their vent fluids' distinct chemistry. Geofluids 8(4):239-251

Liu C-Z, Snow JE, Hellebrand E, Brügmann G, von der Handt A, Büchl A, Hofmann AW (2008) Ancient, highly heterogeneous mantle beneath Gakkel ridge, Arctic Ocean. Nature 452. doi:10.1038/ nature 06688

Mendel V, Sauter D, Patriat P, Munschy M (2000) Evolution of the Central Indian Ridge segmentation with the evolution of the Rodrigues Triple Junction for the past 8 Myr. J Geophys Res 105 (B7):16563-16575

Mével C, Cannat M, Gente P, Marion E, Auzende JM, Karson JA (1991) Emplacement of deep crustal and mantle rocks on the west median valley wall of the MARK area (MAR, $23^{\circ} \mathrm{N} 0$. Tectonophysics 190(1):21-53

Mitchell NC, Escartín J, Allerton S (1998) Detachment faults at midocean ridges garner interest. EOS Trans AGU 79:127

Morishita T, Arai S, Green DH (2003) Evolution of low-Al orthopyroxene in the Horoman peridotite, Japan: an unusual indicator of metasomatising fluids. J Petrol 44(7):1237-1246

Morishita T, Maeda J, Miyashita S, Matsumoto T, Dick HJB (2004) Magmatic srilankite $\left(\mathrm{Ti}_{2} \mathrm{ZrO}_{6}\right)$ in gabbroic vein cutting oceanic peridotites: an unusual product of peridotite-melt interactions beneath slow-spreading ridges. Am Mineral 89(5-6):759-766

Morishita T, Maeda J, Miyashita S, Kumagai H, Matsumoto T, Dick HJB (2007) Petrology of local concentration of chromian spinel in dunite from the slow-spreading Southwest Indian Ridge. Eur J Mineral 19(6):871-882

Morishita T, Hara K, Nakamura K, Sawaguchi T, Tamura A, Arai S, Okino K, Takai K, Kumagai H (2009) Igneous, alteration and exhumation processes recorded in abyssal peridotites and related fault rocks from an oceanic core complex along the Central Indian Ridge. J Petrol 50(7):1299-1325

Nakamura K, Morishita T, Chang Q, Neo N, Kumagai H (2007) Discovery of lanthanide tetrad effect in an oceanic plaiogranite from an Ocean Core Complex at the Central Indian Ridge $25^{\circ} \mathrm{S}$. Geochem J 41(2):135-140

Nakamura K, Morishita T, Bach W, Klein F, Hara K, Okino K, Takai K, Kumagai H (2009) Serpentinized olivine-rich gabbroic rocks exposed near the Kairei Hydrothermal Field, Central Indian Ridge: Insights into the origin of the Kairei hydrothermal fluid supporting a unique microbial ecosystem. Earth Planet Sci Lett 280(1-4):128-136. doi:10.1016/j.eps1.2009.01.024

Niu Y, Hékinian R (1997) Spreading rate dependence of the extent of mantle melting beneath ocean ridges. Nature 385(6614):326-329

Ozawa K, Meyer PS, Bloomer SH (1991) Mineralogy and textures of iron-titanium oxide gabbros and associated olivine gabbros from Hole 735B. Proc Ocean Drill Prog Sci Rep 118:41-73

Parkinson IJ, Pearce JA (1998) Peridotites from the Izu-Bonin-Mariana forearc (ODP Leg 125): evidence for mantle melting and meltmantle interaction in a supra-subduction zone setting. J Petrol 39 (9):1577-1618

Perk NW, Coogan LA, Karson JA, Klein EM, Hanna HD (2007) Petrology and geochemistry of primitice lower oceanic crust from Pito Deep: implications for the accretion of the lower crust at the Southern East Pacific Rise. Contrib Mineral Petrol 154(5):575-590. doi:10.1007/s00410-007-0210-z

Sato T, Okino K, Kumagai H (2009) Magnetic structure of an oceanic core complex at the southernmost Central Indian Ridge: analysis of shipboard and deep-sea three-component magnetometer data. Geochem Geophys Geosyst 10(6). doi:10.1029/ 2008GC002267

Seyler M, Cannat M, Mével C (2003) Evidence for major-element heterogeneity in the mantle source of abyssal peridotites from the Southwest Indian Ridge ( $52^{\circ}$ to $68^{\circ} \mathrm{E}$ ). Geochem Geophys Geosyst 4(2):9101

Seyler M, Loarnd J-P, Dick HJB, Drouin M (2007) Pervasive melt percolatin reactions in ultra-depleted refractory harzburgites at the Mid-Atlantic Ridge, $15^{\circ} 20^{\prime} \mathrm{N}$ : ODP Hole 1274A. Contrib Mineral Petrol 153(3):303-319

Takai K, Gamo T, Tsunogai U (2004) Geochemical and microbiological evidence for a hydrogen-based, hyperthermophilic subsurface lithoautotrophic microbial ecosystem (HyperSLiME) beneath an active deep-sea hydrothermal field. Extremophiles 269-282. doi:10.1007/s00792-004-0386-3

Tamura A, Arai S, Ishimaru S, Andal ES (2008) Petrology and geochemistry of peridotites from IODP Site U1309 at Atlantis Massif, MAR $30^{\circ} \mathrm{N}$ : micro- and macro-scale melt penetrations into peridotites. Contrib Mineral Petrol 155(4):491-509

Van Dover CL (2002) Trophic relationships among invertebrates at the Kairei hydrothermal vent field (Central Indian Ridge). Mar Biol 141:761-772

Van Dover CL, Humphris SE, Fornari D, Cavanaugh CM, Collier R, Goffredi SK, Hashimoto J, Lilley MD, Reysenbach AL, Shank TM, Von Damm KL, Banta A, Gallant RM, Götz D, Green D, Hall J, Harmer TL, Hurtado LA, Johnson P, McKiness ZP, Meredith C, Olsen E, Pan IL, Turnipseed M, Won Y, Young CR III, Verijenhoek RC (2001) Biogeography and ecological setting of Indian Ocean hydrothermal vents. Science 294:818-823

Zanetti A, D'Antonio M, Spadea P, Raffone N, Vannucci R, Brugeir O (2006) Petrogenesis of mantle peridotites from the Izu-BoninMariana (IBM) forearc. Ofioliti 31(2):189-206 